\title{
N-Heterocyclic Carbene Catalysis Exploiting Oxidative Imine Umpolung for the Generation of Imidoyl Azoliums
}

\author{
Tamal Kanti Das, ${ }^{\dagger}, \int$ Krishnaprasad Madica, ${ }^{\ddagger}$ Jagadeesh Krishnan, $₫$ Udaya Kiran \\ Marelli, *,ं, and Akkattu T. Biju*,: \\ ${ }^{\dagger}$ Organic Chemistry Division, CSIR-National Chemical Laboratory, Dr. Homi Bhabha Road, Pune-411008, India \\ ${ }^{\prime}$ Academy of Scientific and Innovative Research (AcSIR), New Delhi 110020, India \\ $\$$ Department of Organic Chemistry, Indian Institute of Science, Bangalore-560012, India
}

\section{Supporting Information}

1. Studies on Enantioselective Version of the Reaction S2

2. X-Ray Data of $\mathbf{3 y} \quad$ S3

3. Mechanistic Experiments S5

4. Natural Population Analysis (NPA) S13

5. ${ }^{1} \mathrm{H}$ and ${ }^{13} \mathrm{C}$ NMR Spectra of 3,1-Benzoxazine Derivatives $\quad \mathrm{S} 15$

6. Cartesian Coordinates for the Optimized Structures S45 


\section{Studies on Enantioselective Version of the Reaction}

\section{2-(4-Nitrophenyl)-4-(trifluoromethyl)-4H-benzo $[d][1,3]$ oxazine (chiral-3a)}

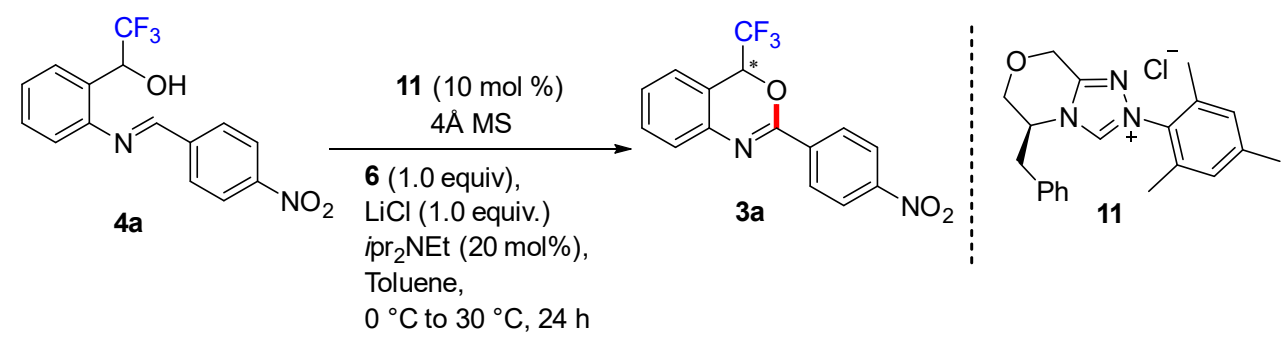

\section{HPLC Spectra:}

Sampl e I nf o : CH RALCEL- OD- H, 2\% I PA-Hexane, $1.0 \mathrm{~mL} / \mathrm{m} \mathrm{n}, 254 \mathrm{~nm}$

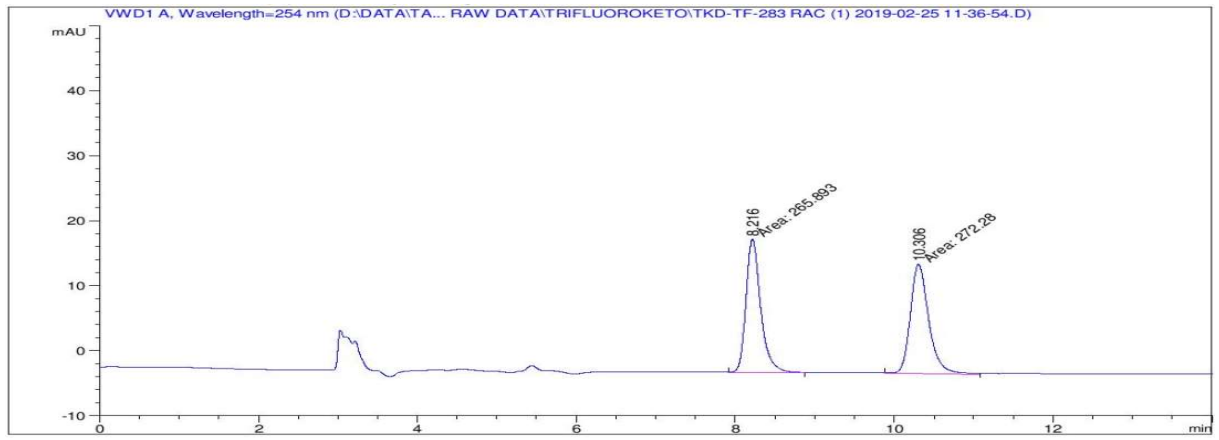

\begin{tabular}{|c|c|c|c|c|c|c|}
\hline eak & $\begin{array}{l}\text { Ret Ti me } \\
{[\mathrm{mi} \mathrm{n}]}\end{array}$ & Type & $\begin{array}{l}W \mathrm{dth} \\
{[\mathrm{m} \mathrm{n}]}\end{array}$ & $\begin{array}{c}\text { Area } \\
{\left[\mathrm{mAU}^{*} \mathrm{~s}\right]}\end{array}$ & $\begin{array}{l}\text { Hei ght } \\
\text { [ } \mathrm{mAU}]\end{array}$ & $\begin{array}{c}\text { Area } \\
\%\end{array}$ \\
\hline & & & & & & \\
\hline & 0.306 & & 2693 & 272. & 6. 85310 & 93 \\
\hline
\end{tabular}

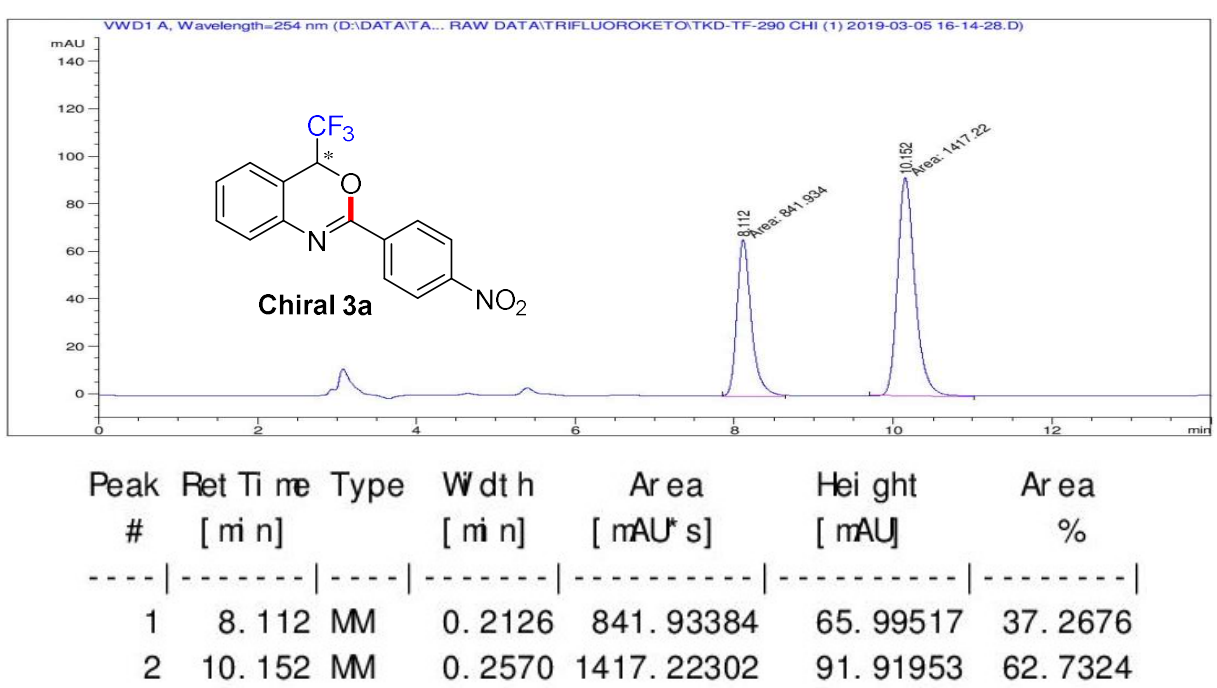


4-Methyl-2-(4-nitrophenyl)-4-(trifluoromethyl)-4H-benzo[d][1,3]oxazine (chiral 3ac)

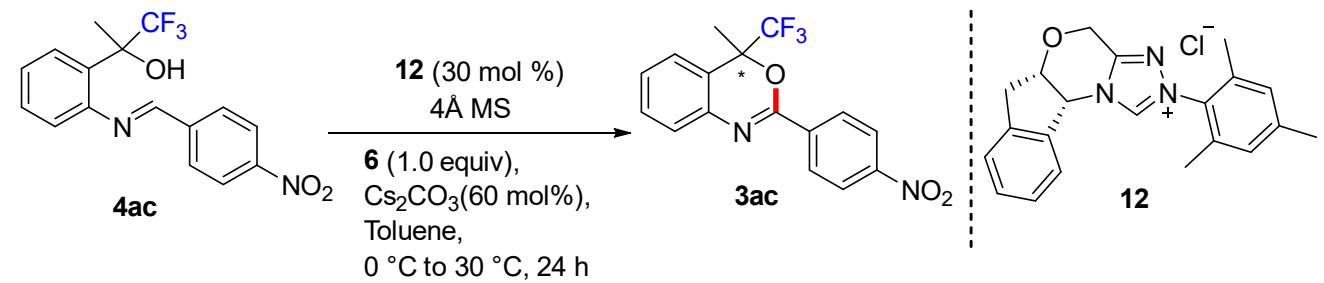

\section{HPLC Spectra:}

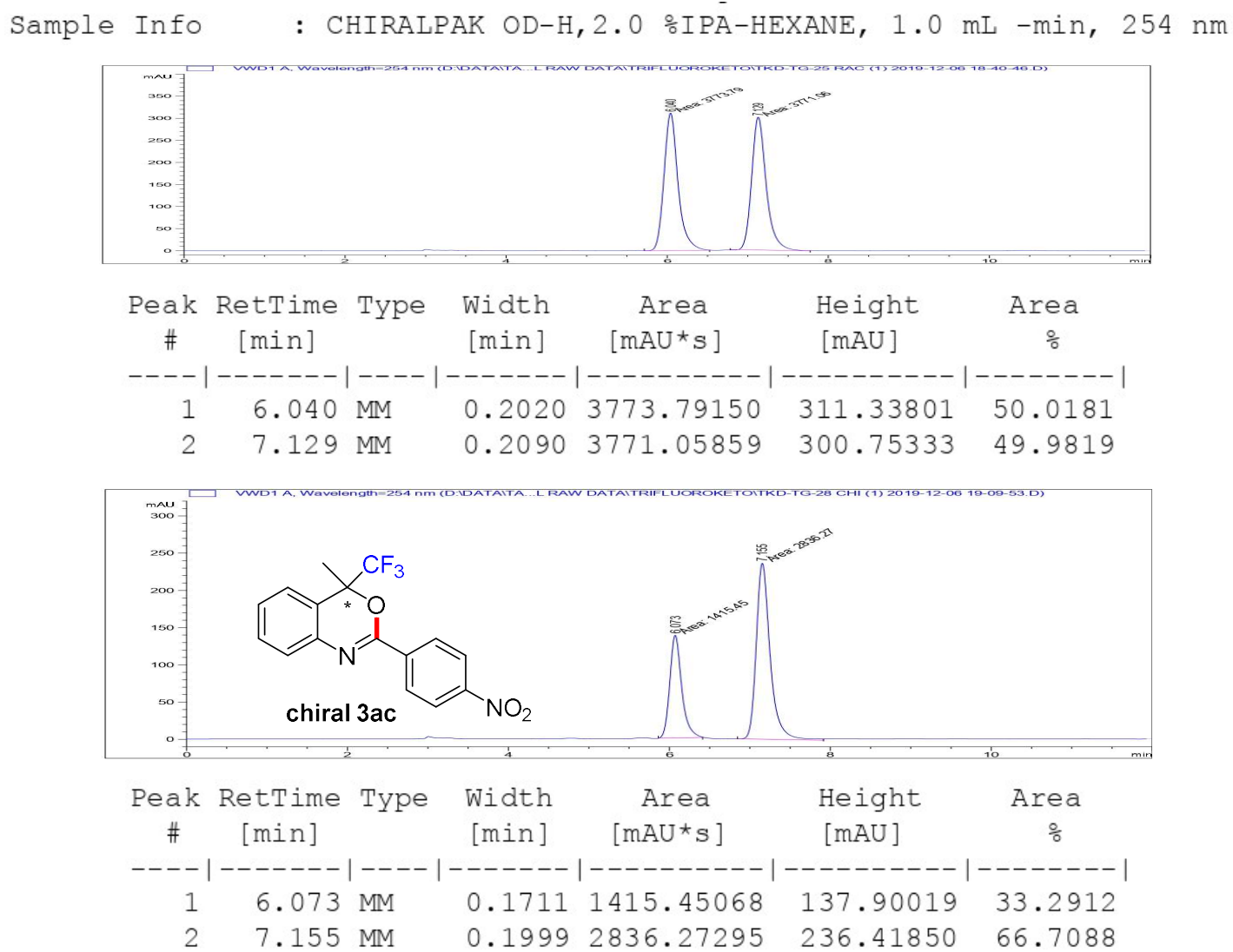

\section{X-Ray Data of $3 y$}

Single crystal of $\mathbf{3 y}$ (recrystallized from $1: 4 \mathrm{CHCl}_{3} / n$-hexane at $30{ }^{\circ} \mathrm{C}$ ) was mounted and the diffraction data was collected at $296 \mathrm{~K}$ on a Bruker APEX-II CCD diffractometer using SMART/SAINT software. Intensity data were collected using MoK $\alpha$ radiation $\left(\lambda=0.71073 \mathrm{~A}^{\circ}\right)$. The single crystal was affixed to a Hampton Research cryoloop using Paratone-N oil. Data collection and reduction was performed using Bruker APEX2 and Bruker SAINT, respectively. The structure was solved by direct methods using the SHELX-97 and refined by full-matrix least-squares on F2. Empirical absorption corrections were applied with SADABS. All Non- 
hydrogen atoms were refined anisotropically and hydrogen atoms were included in geometric positions. Structure was drawn using Olex-2 and Mercury-3. The crystallographic refinement parameters are given below:

\begin{tabular}{|c|c|}
\hline Identification code & $3 y$ \\
\hline CCDC & 1941009 \\
\hline Empirical formula & $\mathrm{C}_{24} \mathrm{H}_{14} \mathrm{~F}_{6} \mathrm{~N}_{2} \mathrm{O}_{2}$ \\
\hline Formula weight & 476.37 \\
\hline Temperature/K & $296(2)$ \\
\hline Crystal system & triclinic \\
\hline Space group & $\mathrm{P}-1$ \\
\hline $\mathrm{a} / \AA$ & $5.7211(4)$ \\
\hline $\mathrm{b} / \AA$ & $8.2943(6)$ \\
\hline $\mathrm{c} / \AA$ & $11.0522(7)$ \\
\hline$\alpha /{ }^{\circ}$ & $92.866(4)$ \\
\hline$\beta / \circ$ & $91.401(4)$ \\
\hline$\gamma /{ }^{\circ}$ & $99.979(4)$ \\
\hline Volume $/ \AA^{3}$ & $515.58(6)$ \\
\hline $\mathrm{Z}$ & 1 \\
\hline$\rho_{\text {calc }} \mathrm{g} / \mathrm{cm}^{3}$ & 1.534 \\
\hline$\mu / \mathrm{mm}^{-1}$ & 0.135 \\
\hline $\mathrm{F}(000)$ & 242.0 \\
\hline Crystal size $/ \mathrm{mm}^{3}$ & $0.251 \times 0.233 \times 0.244$ \\
\hline Radiation & $\operatorname{MoK} \alpha(\lambda=0.71073)$ \\
\hline \multicolumn{2}{|c|}{$2 \Theta$ range for data collection $/{ }^{\circ} 3.692$ to 54.994} \\
\hline Index ranges & $-7 \leq \mathrm{h} \leq 7,-10 \leq \mathrm{k} \leq 10,-13 \leq 1 \leq 14$ \\
\hline Reflections collected & 8046 \\
\hline Independent reflections & $2307\left[\mathrm{R}_{\mathrm{int}}=0.0247, \mathrm{R}_{\text {sigma }}=0.0269\right]$ \\
\hline Data/restraints/parameters & $2307 / 0 / 158$ \\
\hline Goodness-of-fit on $\mathrm{F}^{2}$ & 1.030 \\
\hline Final $R$ indexes $[I>=2 \sigma(I)]$ & $\mathrm{R}_{1}=0.0410, \mathrm{wR}_{2}=0.1031$ \\
\hline Final $\mathrm{R}$ indexes [all data] & $\mathrm{R}_{1}=0.0706, \mathrm{wR}_{2}=0.1193$ \\
\hline Largest diff. peak/hole / e $\AA$ & $0.15 /-0.18$ \\
\hline
\end{tabular}

CCDC 1941009 (3y) contains the supplementary crystallographic data for this paper. These data can be obtained free of charge from Data Centre via www.ccdc.cam.ac.uk/data_request/cif. 


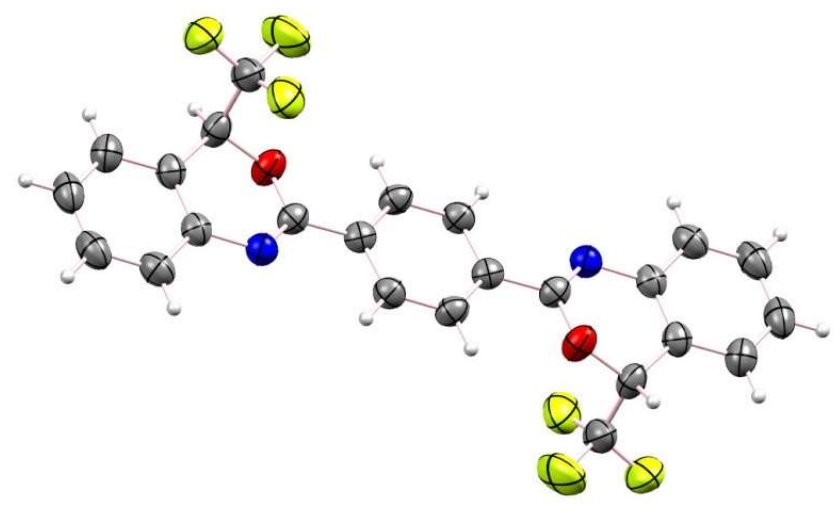

Figure S1: ORTEP diagram of $\mathbf{3 y}$ (thermal ellipsoids at 50\% probability)

\section{Mechanistic Experiments}

a) Synthesis of 2-phenyl-4H-benzo[d][1,3]oxazine

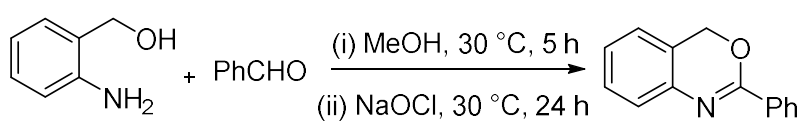

Following the literature procedure ${ }^{1}$, a solution of 2-amino-benzylalcohol (31.0 $\left.\mathrm{mg}, 0.25 \mathrm{mmol}\right)$ and benzaldehyde $(25 \mu \mathrm{L}, 0.25 \mathrm{mmol})$ in $1.0 \mathrm{~mL}$ of dry methanol was stirred at room temperature $\left(30{ }^{\circ} \mathrm{C}\right.$ ) for 5 hours, followed by addition of $250 \mu \mathrm{L}$ of $\mathrm{NaOCl}(4 \%$ available chlorine; 3 equiv) drop wise and the reaction mixture was stirred at room temperature for $12 \mathrm{~h}$. The solvent was evaporated under reduced pressure, and the residue was mixed with water and extracted with ethyl acetate. The extract was washed with brine, dried over $\mathrm{Na}_{2} \mathrm{SO}_{4}$ and solvent was removed. The product 2-phenyl$4 H$-benzo $[d][1,3]$ oxazine was isolated by flash column chromatography as white solid $(43.0 \mathrm{mg}, 82$ $\%$ yield). The NMR data was in accordance with the literature report.

${ }^{1}$ Kumar, R. A.; Maheswari, C. U.; Ghantasala, S.; Jyothi, C.; Reddy, K. R. Adv. Synth. Catal. 2011, 353, 401. 


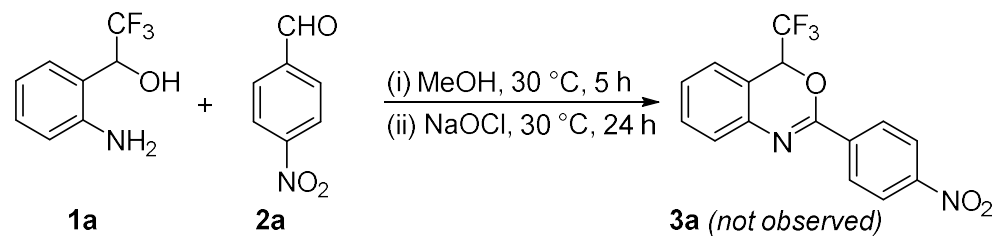

Following the same literature procedure ${ }^{2}$, a solution of 1-(2-aminophenyl)-2,2,2trifluoroethan-1-ol 1a $(48.0 \mathrm{mg}, 0.25 \mathrm{mmol})$ and 4-nitrobenzaldehyde $\mathbf{2 a}(38.0 \mathrm{mg}, 0.25 \mathrm{mmol})$ in $1.0 \mathrm{~mL}$ of dry methanol was stirred at room temperature for 5 hours, followed by addition of $250 \mu \mathrm{L}$ of $\mathrm{NaOCl}$ (4\% available chlorine; 3 equiv) drop-wise and the reaction mixture was stirred at room temperature for $24 \mathrm{~h}$. The reaction did not afford expected product $3 \mathbf{a}$.

These studies shed light on the absence of dihydro 3,1-benzoxazine formation and possibly the role of NHC in the intramolecular cyclization leading to the formation of 3,1-benzoxazines.

\section{b) Synthesis of trifluoromethylated 3,1-benzoxazine derivative 3 a from imine 4 a}

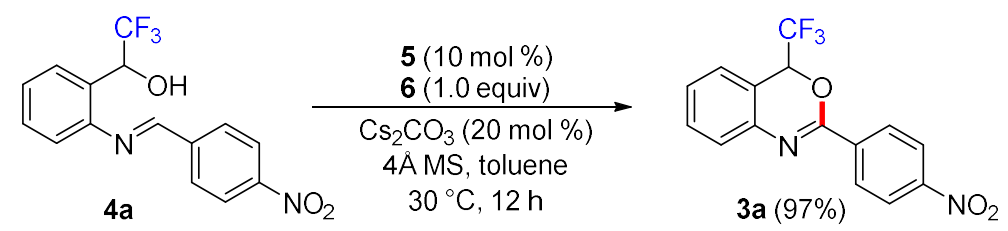

The [(E)-2,2,2-trifluoro-1-(2-((4-nitrobenzylidene)amino)phenyl)ethan-1-ol] 4a (purified by recrystallization) $(162.0 \mathrm{mg}, 0.5 \mathrm{mmol})$ was added to a solution of triazolium salt $5(13.6 \mathrm{mg}$, $0.05 \mathrm{mmol})$ and $\mathrm{Cs}_{2} \mathrm{CO}_{3}(32.5 \mathrm{mg}, 0.10 \mathrm{mmol})$ in dry toluene $(4.0 \mathrm{~mL})$ in a screw-capped test tube equipped with a magnetic stir bar under argon atmosphere. To this mixture was added oxidant 6 (204.0 mg, $0.50 \mathrm{mmol})$ and $4 \AA \mathrm{MS}(100 \mathrm{mg})$ and it was stirred at $30^{\circ} \mathrm{C}$ for $12 \mathrm{~h}$. The reaction mixture was then filtered, and the solvent was evaporated to get the crude residue, which was purified by flash column chromatography (Pet.ether-EtOAc: 95:5) on silica gel to afford 2-(4-nitrophenyl)-4-(trifluoromethyl)-4H-benzo[d][1,3]oxazine 3a as a pale yellow solid (156.0 mg, 97\% yield).

This reaction indicates the intermediacy of aldimine $4 \boldsymbol{a}$ in the annulation reaction. 
c) Reaction with $1 a$ and $2 a$ without allowing in-situ imine formation

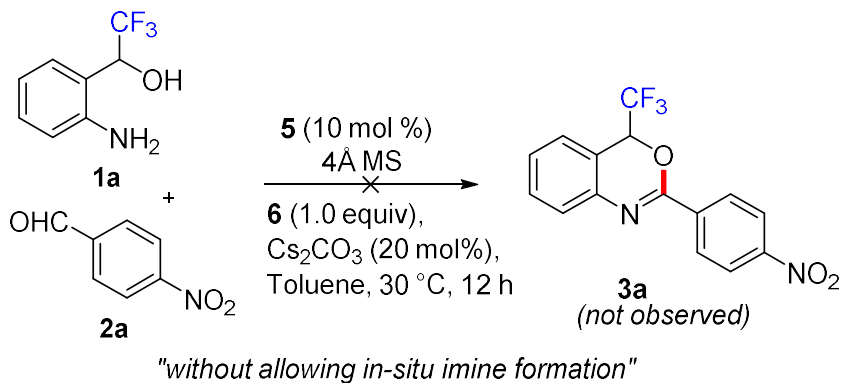

The 1-(2-aminophenyl)-2,2,2-trifluoroethan-1-ol $\mathbf{1 a}(48.0 \mathrm{mg}, \quad 0.25 \mathrm{mmol})$ and 4nitrobenzaldehyde $2 \mathbf{2 a}(38.0 \mathrm{mg}, 0.25 \mathrm{mmol})$ was treated with triazolium salt $\mathbf{5}(6.8 \mathrm{mg}, 0.025$ $\mathrm{mmol}), \mathrm{Cs}_{2} \mathrm{CO}_{3}(16.3 \mathrm{mg}, 0.05 \mathrm{mmol})$, oxidant $6(102.0 \mathrm{mg}, 0.25 \mathrm{mmol})$ and $4 \AA \mathrm{MS}(50.0 \mathrm{mg})$ in toluene $(2.0 \mathrm{~mL})$ at $30{ }^{\circ} \mathrm{C}$ for $12 \mathrm{~h}$. The reaction mixture was then filtered and concentrated to obtain crude residue. Under this reaction condition product 3a was not formed.

The lack of product $3 \boldsymbol{a}$ formation indicates that the aldimine $4 \boldsymbol{a}$ was not generated in situ under these conditions.

\section{d) Control experiments and optimization of $\mathrm{NHC}$-catalyzed reaction}
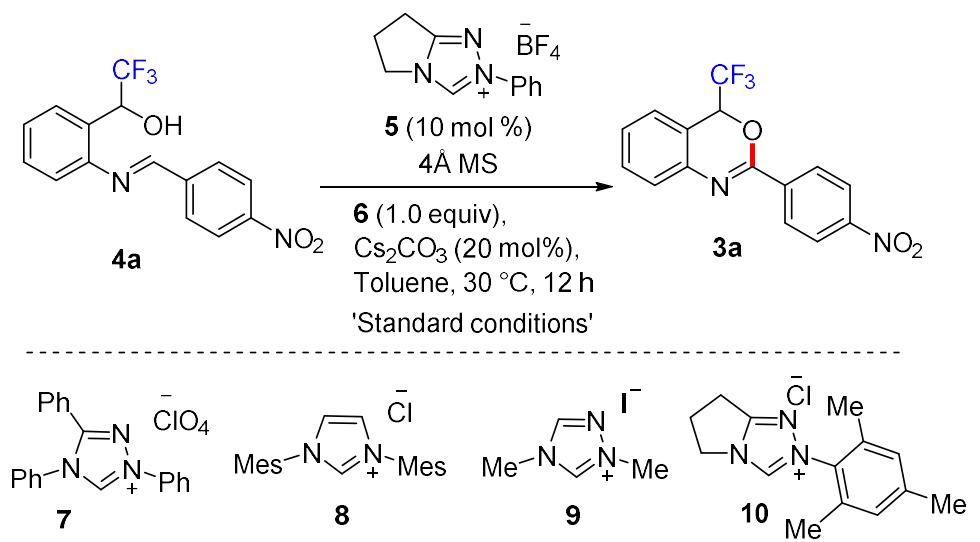

\begin{tabular}{|c|l|c|}
\hline entry & \multicolumn{1}{|c|}{ variation of standard conditions $^{\mathrm{a}}$} & $\begin{array}{c}\text { yield of 3a } \\
(\mathbf{\%})^{\mathrm{b}}\end{array}$ \\
\hline 1 & none & $99(97)$ \\
\hline 2 & without $\mathbf{5}$ & $<5$ \\
\hline 3 & $\begin{array}{l}\text { without } \mathbf{5}, 100 \mathrm{~mol} \% \mathrm{Cs}_{2} \mathrm{CO}_{3} \text { instead } \\
20 \text { mol \% }\end{array}$ & $<5$ \\
\hline 4 & air as an oxidant & 70 \\
\hline 5 & 5 mol \% 5 instead of 10 mol \% & 87 \\
\hline 6 & $\mathbf{7}$ instead of $\mathbf{5}$ & 95 \\
\hline
\end{tabular}




\begin{tabular}{|c|l|c|}
\hline 7 & $\mathbf{8}$ instead of $\mathbf{5}$ & 96 \\
\hline 8 & $\mathbf{9}$ instead of $\mathbf{5}$ & 72 \\
\hline 9 & $\mathbf{1 0}$ instead of $\mathbf{5}$ & 94 \\
\hline 10 & $\mathrm{KO} t$-Bu instead of $\mathrm{Cs}_{2} \mathrm{CO}_{3}$ & 82 \\
\hline 11 & DBU instead of $\mathrm{Cs}_{2} \mathrm{CO}_{3}$ & 97 \\
\hline 12 & $i$-Pr 2 EtN instead of $\mathrm{Cs}_{2} \mathrm{CO}_{3}$ & $<5$ \\
\hline 13 & DMF instead of toluene & 99 \\
\hline 14 & THF instead of toluene & 82 \\
\hline 15 & DCM instead of toluene & 96 \\
\hline
\end{tabular}

a Standard conditions: $4 \mathrm{a}(0.25 \mathrm{mmol}), 5(0.025 \mathrm{mmol}), \mathrm{Cs}_{2} \mathrm{CO}_{3}(0.05 \mathrm{mmol}), 6$ (0.25 mmol), toluene $(2.0 \mathrm{~mL}), 30{ }^{\circ} \mathrm{C}, 12 \mathrm{~h} .{ }^{\mathrm{b}}$ The yields were determined by ${ }^{1} \mathrm{H}$ NMR analysis of crude product using $\mathrm{CH}_{2} \mathrm{Br}_{2}$ as the internal standard.

\section{e) Reaction with varying catalyst loading}

5 Sets of parallel experiments were carried out by treating the aldimine 4a (16.2 $\mathrm{mg}, 0.05$ mmol) with triazolium salt 5 (x mol \%), $\mathrm{Cs}_{2} \mathrm{CO}_{3}(2 \mathrm{x}$ mol \%), oxidant 6 (20.4 mg, $0.05 \mathrm{mmol})$ in toluene $(0.5 \mathrm{~mL})$ at $30{ }^{\circ} \mathrm{C}$ for $0.5 \mathrm{~h}$. The reaction mixture was then passed through a short silica pad and concentrated to obtain crude residue, which was analyzed by ${ }^{1} \mathrm{H}$ NMR analysis of crude product using 1,3,5-trimethoxy benzene as the internal standard.

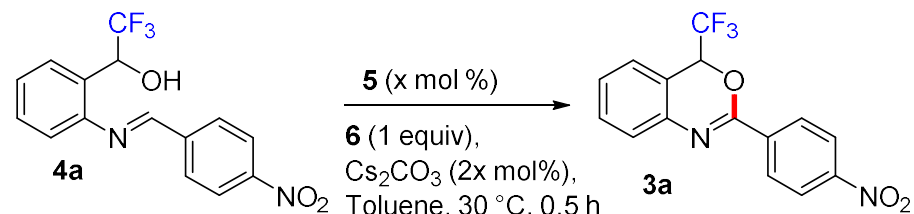

\begin{tabular}{|c|c|c|c|}
\hline run & triazolium salt 5 & $\mathrm{Cs}_{2} \mathrm{CO}_{3}$ & Yield 3a (\%) \\
\hline 1 & $0 \mathrm{~mol} \%$ & $0 \mathrm{~mol} \%$ & 0 \\
\hline 2 & $5 \mathrm{~mol} \%$ & $10 \mathrm{~mol} \%$ & 26 \\
\hline 3 & $10 \mathrm{~mol} \%$ & $20 \mathrm{~mol} \%$ & 38 \\
\hline 4 & $15 \mathrm{~mol} \%$ & $30 \mathrm{~mol} \%$ & 61 \\
\hline 5 & $20 \mathrm{~mol} \%$ & $40 \mathrm{~mol} \%$ & 80 \\
\hline
\end{tabular}




\section{${ }^{1} \mathrm{H}$ NMR spectra of the crude reaction mixture}
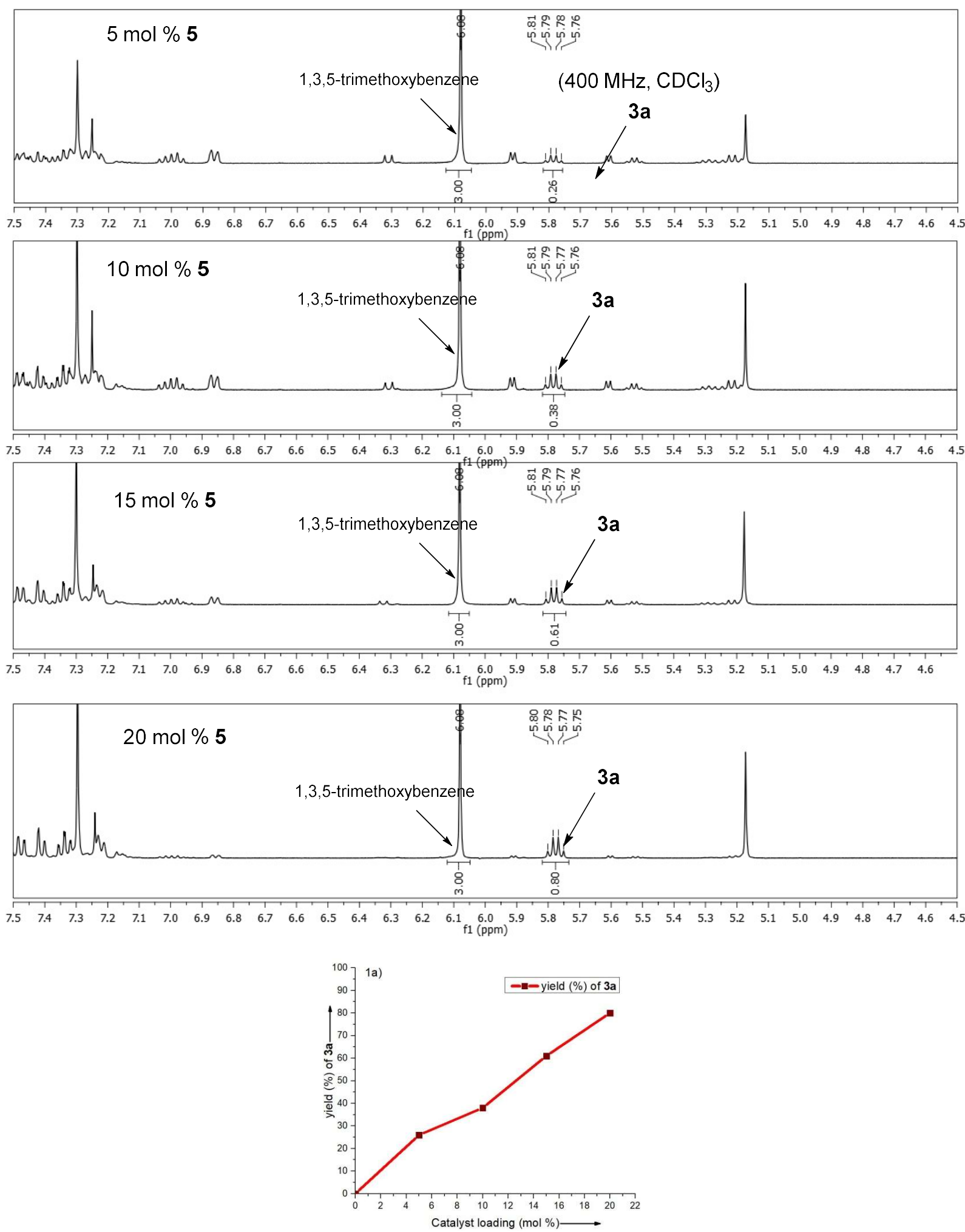

Figure S2: Yield (\%) of 3a vs catalyst loading 


\section{f) Kinetic studies for the determination of reaction order ${ }^{2}$}

The aldimine [(E)-2,2,2-trifluoro-1-(2-((4-nitrobenzylidene)amino)phenyl)ethan-1-ol] $4 \mathbf{a}$ (16.2 mg, $0.05 \mathrm{mmol})$ was treated with triazolium salt $5(1.4 \mathrm{mg}, 0.005 \mathrm{mmol}), \mathrm{Cs}_{2} \mathrm{CO}_{3}(3.2 \mathrm{mg}$, $0.01 \mathrm{mmol})$, oxidant $6(20.4 \mathrm{mg}, 0.05 \mathrm{mmol})$ and $4 \AA \mathrm{MS}(10 \mathrm{mg})$ in toluene $(0.5 \mathrm{~mL})$ at $30{ }^{\circ} \mathrm{C}$ for definite time. The reaction mixture was then filtered and concentrated to obtain crude residue, which was analyzed using ${ }^{1} \mathrm{H}$ NMR. 1,3,5-trimethoxy benzene $(8.4 \mathrm{mg}, 0.05 \mathrm{mmol})$ was used as the internal standard.

\begin{tabular}{|l|l|l|l|l|}
\hline Experiment & Substrate 4a & $\begin{array}{l}\text { Carbene } \\
\text { Precursor 5 }\end{array}$ & $\mathrm{Cs}_{2} \mathrm{CO}_{3}$ & Oxidant 6 \\
\hline Run 1 & $0.05 \mathrm{mmol}$ & $0.005 \mathrm{mmol}$ & 0.01 & $0.05 \mathrm{mmol}$ \\
\hline Run 2 & $0.10 \mathrm{mmol}$ & $0.005 \mathrm{mmol}$ & 0.01 & $0.10 \mathrm{mmol}$ \\
\hline
\end{tabular}
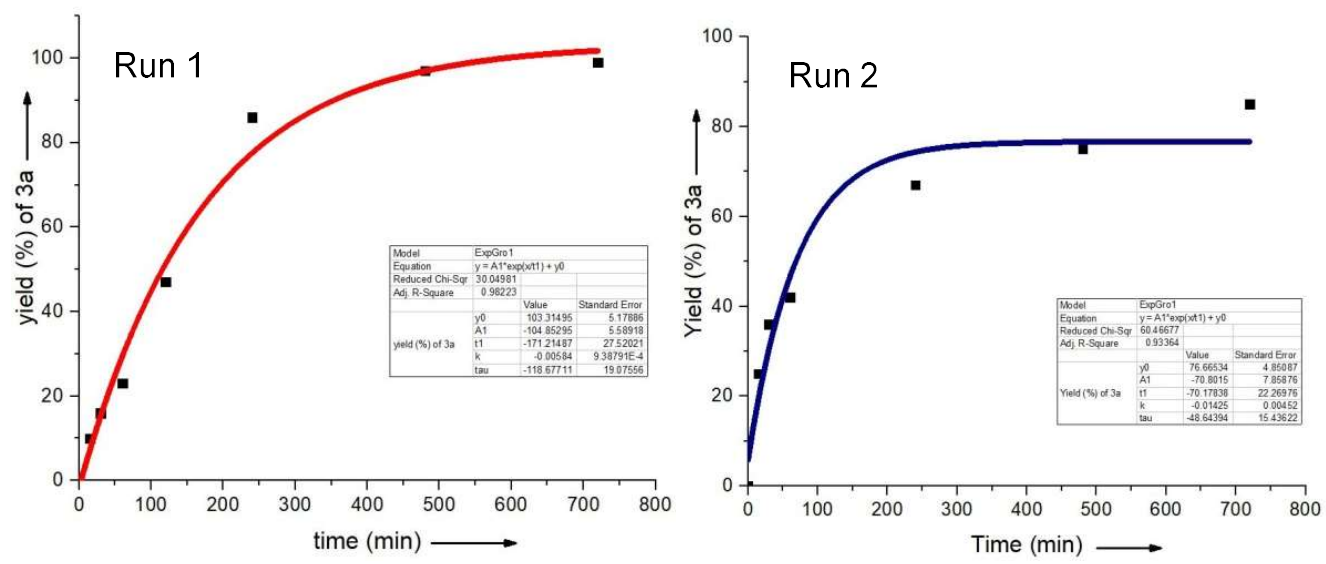

Figure S3: Yield (\%) of 3a vs time

\section{Run 1:}

Rate $_{\text {Run } 1}=\mathrm{dy} / \mathrm{dx}=\left(\mathrm{y}_{2}-\mathrm{y}_{1}\right) /\left(\mathrm{x}_{2}-\mathrm{x}_{1}\right)=0.00889$

Rate $_{\text {Run } 1}=0.00889=\mathrm{k}[\text { Substrate }]^{\mathrm{n}}$

\section{Run 2:}

Rate $_{\text {Run2 }}=\mathrm{dy} / \mathrm{dx}=\left(\mathrm{y}_{2}-\mathrm{y}_{1}\right) /\left(\mathrm{x}_{2}-\mathrm{x}_{1}\right)=0.02$

Rate $_{\text {Run2 }}=0.02=\mathrm{k}[\text { Substrate }]^{\mathrm{n}}$

Hence from equation (i) and (ii)

Rate $_{\text {Run2 }} /$ Rate $_{\text {Run } 1}=\mathrm{k}[0.10]^{\mathrm{n}} / \mathrm{k}[0.05]^{\mathrm{n}}$

${ }^{2}$ Bera, M.; Agasti, S.; Chowdhury, R.; Mondal, R.; Pal, D.; Maiti, D. Angew. Chem. Int. Ed. 2017, 56, $5272-5276$. 
$(0.02 / 0.00889)=2^{\mathrm{n}}$

$\log (0.02 / 0.00889)=\mathrm{n} \log 2$

$\mathrm{n}=0.352 / 0.301$

$\mathrm{n}=1.169 \sim 1.0$

\section{Order with respect to substrate imine (4a) is 1}

Now, Rate Constant k $=(0.00889) /(0.05)^{1} \mathrm{sec}^{-1}$

$$
\mathrm{k}=0.1778 \mathrm{sec}^{-1}
$$

\section{g) Intermolecular Competition Experiment}

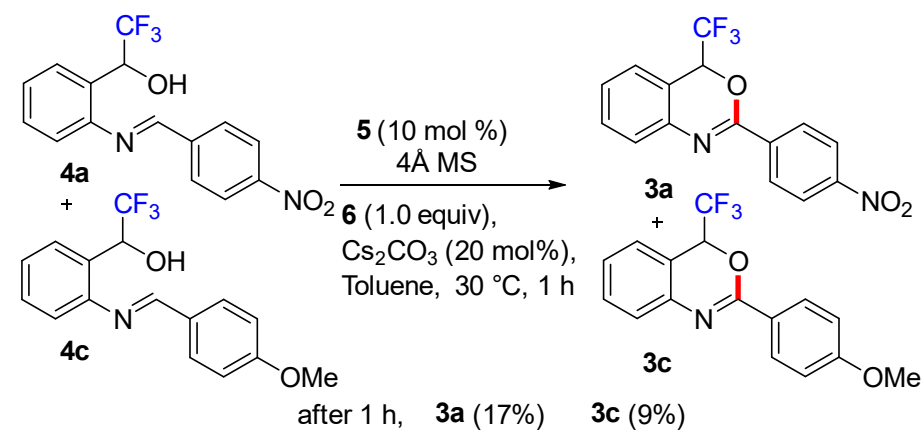

The aldimine $4 \mathrm{a}(40.5 \mathrm{mg}, 0.125 \mathrm{mmol})$ and $4 \mathrm{c}(38.5 \mathrm{mg}, 0.125 \mathrm{mmol})$ were treated with triazolium salt 5 (6.8 mg, $0.025 \mathrm{mmol}), \mathrm{Cs}_{2} \mathrm{CO}_{3}(16.3 \mathrm{mg}, 0.05 \mathrm{mmol})$, oxidant 6 (102.0 mg, 0.25 $\mathrm{mmol})$ and $4 \AA \mathrm{MS}(50.0 \mathrm{mg})$ in toluene $(2.0 \mathrm{~mL})$ at $30{ }^{\circ} \mathrm{C}$ for $1 \mathrm{~h}$. The reaction mixture was then filtered and concentrated to obtain crude residue, which was analyzed using ${ }^{1} \mathrm{H}$ NMR. $\mathrm{CH}_{2} \mathrm{Br}_{2}(18.0 \mu \mathrm{L}, 0.25 \mathrm{mmol})$ was used as the internal standard.

This reaction shows that electron-deficient aldimine 4 a reacted $\sim 2$ times faster than the electronrich one $4 c$.

\section{${ }^{1} \mathrm{H} N M R$ of the crude reaction mixture}

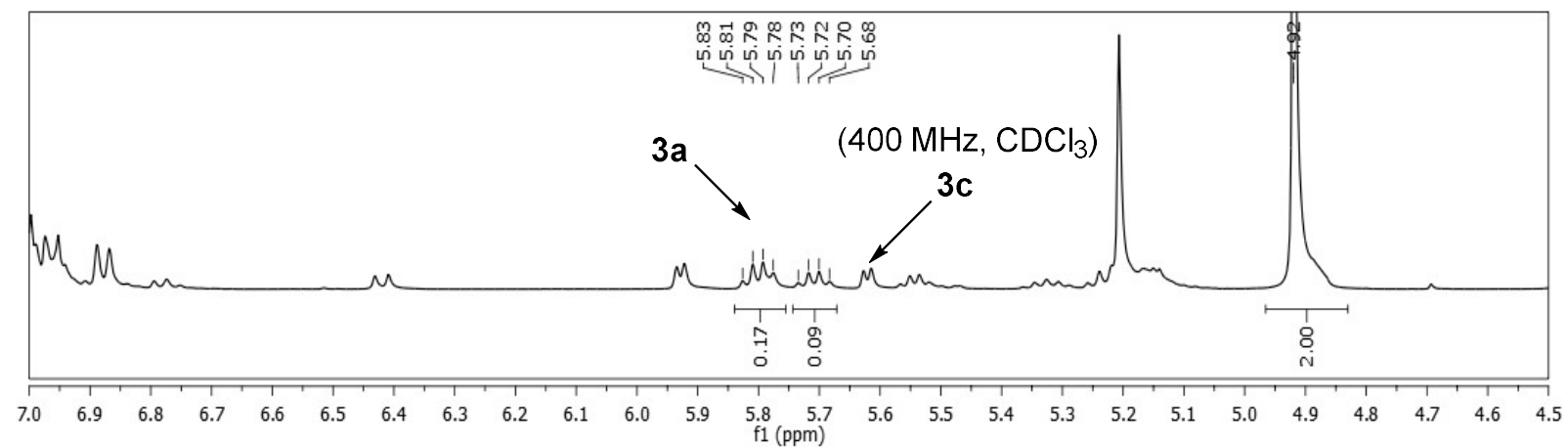


${ }^{1} \mathrm{H} N M R$ of $3 a$ and $3 c$
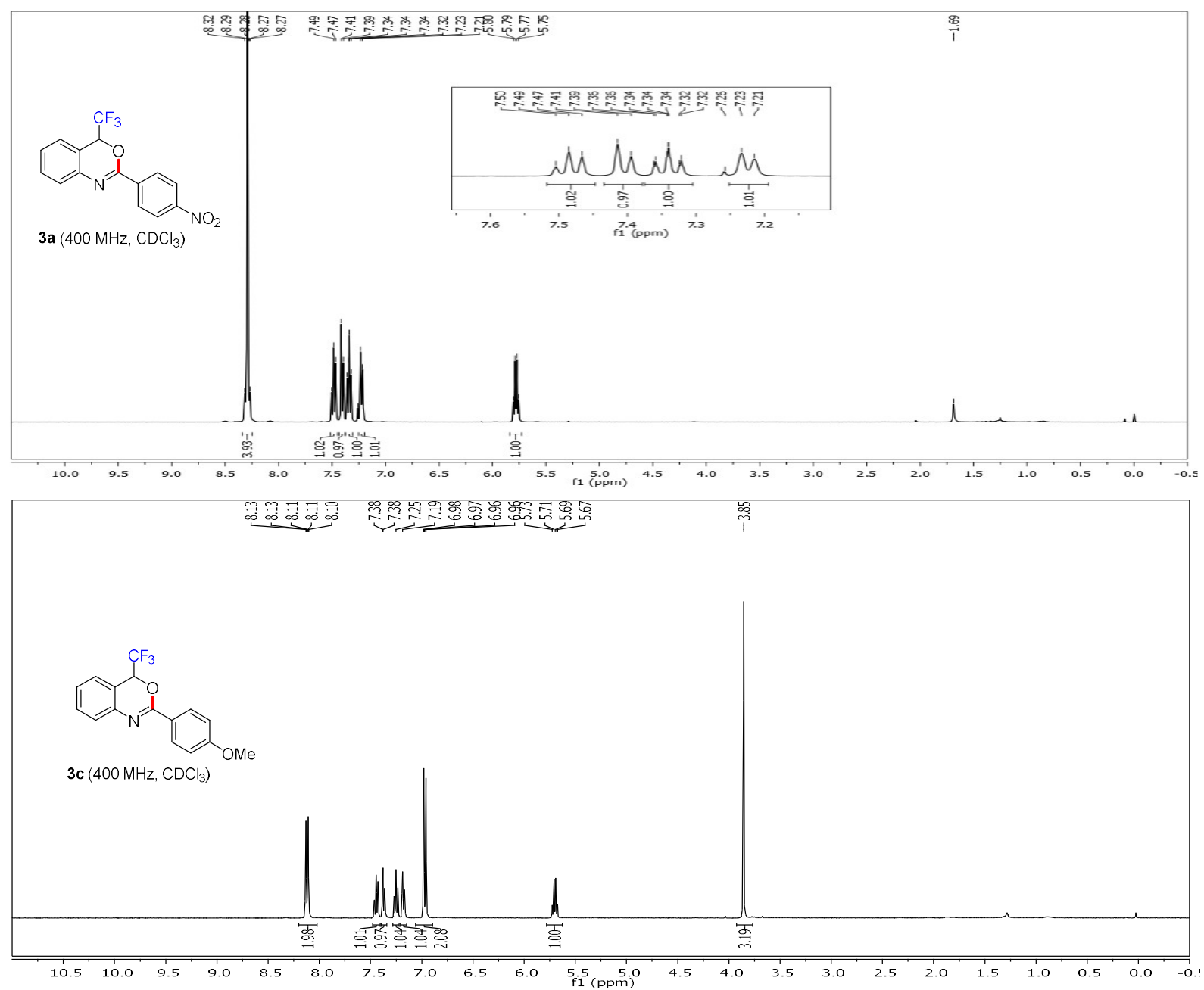

h) Experiment to rule out the role of $\mathrm{NHC}$ as Bronsted base

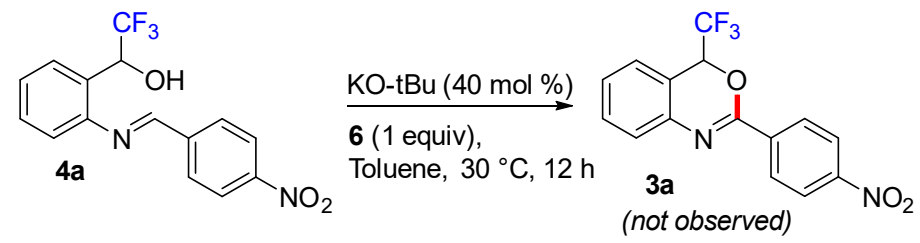

A reaction was carried out by treating the pre-formed imine $4 \mathbf{a}(0.25 \mathrm{mmol})$ with $\mathrm{KO}-\mathrm{t} \mathrm{Bu}$ (11.0 mg, $0.1 \mathrm{mmol})$, oxidant $6(102.0 \mathrm{mg}, 0.25 \mathrm{mmol})$ and $4 \AA \mathrm{MS}(50.0 \mathrm{mg})$ in toluene (2.0 $\mathrm{mL}$ ) at $30^{\circ} \mathrm{C}$ for $12 \mathrm{~h}$. Under this reaction condition expected product 3a was not observed.

This reaction proves that NHC is not acting as Brønsted base in the present annulation reaction. 


\section{Natural Population Analysis (NPA):}

\section{Computational Details}

All electronic structure and frequency calculations were performed using Gaussian ${ }^{3}$ suite of quantum chemistry program. Geometries of all the species involved have been optimized by employing Density Functional Theory (DFT). All the geometries were optimized with M06-2X method $^{4}$ in conjunction with $6-31++\mathrm{G}(\mathrm{d}, \mathrm{p})$ basis set. The solvent effect was considered by the implementation of conductor-like screening solvation model (COSMO $)^{5}$ considering toluene as the bulk solvent medium.

\section{Fukui functions}

In order to compare the nucleophilicity and electrophilicity, NPA Charge, electrophilic $\mathrm{f}_{\mathrm{k}}^{+}$ and nucleophilic $\mathrm{f}_{\mathrm{k}}{ }^{-}$condensed Fukui functions was calculated. Natural population analysis (NPA) of the imidoyl azolium intermediate $\mathbf{A}$ gives charge of +0.255 on carbon atom $\mathrm{C} 2$, indicating it to be a electrophilic site, while -0.786 on $\mathrm{O} 2$, specifying it to be nucleophilic. As depicted in Table S1, higher value of electrophilic Fukui function $\left(\mathrm{f}_{\mathrm{k}}^{+}\right)$for imidoyl azolium intermediate $\mathbf{A}$ in comparison to imine 4a indicates $\mathrm{C} 2$ to be more electrophilic than $\mathrm{C} 1$ of imine 4a. Similarly, the nucleophilic Fukui function $\left(\mathrm{f}_{\mathrm{k}}{ }^{-}\right)$on $\mathrm{O} 2$ is found to be higher for imidoyl azolium intermediate A compared to imine 4a, projecting it to be more nuclophilic.

$$
\begin{array}{ll}
f_{k}^{+}=\left[q_{k}(N+1)-q_{k}(N)\right] & \text { (for nucleophilic attack) } \\
f_{k}^{-}=\left[q_{k}(N)-q_{k}(N-1)\right] & \text { (for electrophilic attack) } \\
f_{k}^{+} \text {and } f_{k}^{-} \text {are respectively the electrophilic and nucleophilic Fukui functions. }
\end{array}
$$

\footnotetext{
${ }^{3}$ Frisch, M. J.; Trucks, G. W.; Schlegel, H. B.; Scuseria, G. E.; Robb, M. A.; Cheeseman, J. R.; Scalmani, G.; Barone, V.; Mennucci, B.; Petersson, G. A.; Nakatsuji, H.; Caricato, M.; Li, X.; Hratchian, H. P.; Izmaylov, A. F.; Bloino, J.; Zheng, G.; Sonnenberg, J. L.; Hada, M.; Ehara, M.; Toyota, K.; Fukuda, R.; Hasegawa, J.; Ishida, M.; Nakajima, T.; Honda, Y.; Kitao, O.; Nakai, H.; Vreven, T.; Montgomery, J. A. Jr.; Peralta, J. E.; Ogliaro, F.; Bearpark, M. J.; Heyd, J.; Brothers, E. N.; Kudin, K. N.; Staroverov, V. N.; Kobayashi, R.; Normand, J.; Raghavachari, K.; Rendell, A. P.; Burant, J. C.; Iyengar, S. S.; Tomasi, J.; Cossi, M.; Rega, N.; Millam, N. J.; Klene, M.; Knox, J. E.; Cross, J. B.; Bakken, V.; Adamo, C.; Jaramillo, J.; Gomperts, R.; Stratmann, R. E.; Yazyev, O.; Austin, A. J.; Cammi, R.; Pomelli, C.; Ochterski, J. W.; Martin, R. L.; Morokuma, K.; Zakrzewski, V. G.; Voth, G. A.; Salvador, P.; Dannenberg, J. J.; Dapprich, S.; Daniels, A. D.; Farkas, Ö.; Foresman, J. B.; Ortiz, J. V.; Cioslowski, J.; Fox, D. J. 2013, Gaussian 09, Revision D.01, Gaussian, Inc., Wallingford, CT, USA.

${ }^{4}$ Zhao, Y.; Truhlar, D. G. Theor. Chem. Acc. 2008, 120, 215.

${ }^{5}$ Klamt, A.; Schüürmann, G. J. Chem. Soc., Perkin Trans. 2. 1993, 799.
} 

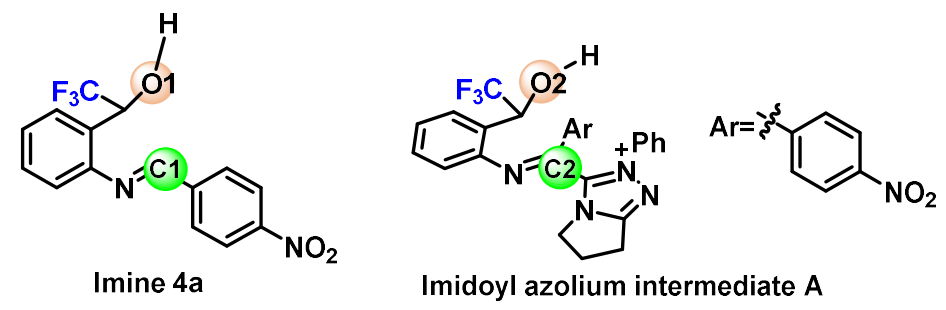

There is no negative frequency involved as it is not a transition state, but an intermediate. Only a transition state is a $1^{\text {st }}$ order saddle point with one negative frequency.

The theoretically calculated energy values for imine $\mathbf{4 a}$ and intermediate $\mathbf{A}$ is provided below:

Imine 4a: -1212.6 Hartree

Intermediate A: -1801.6 Hartree

\begin{tabular}{|c|c|c|c|}
\hline Atom & NPA & $\mathbf{f}_{\mathbf{k}}{ }^{+}$ & $\mathbf{f}_{\mathbf{k}}{ }^{-}$ \\
\hline $\mathrm{C}(1)$ & 0.135 & 0.05 & \\
$\mathrm{C}(2)$ & 0.255 & 0.25 & \\
$\mathrm{O}(1)$ & -0.734 & & -0.04 \\
$\mathrm{O}(2)$ & -0.786 & & 0.02 \\
\hline
\end{tabular}

Table S1: NPA charge, electrophilic $\mathrm{f}_{\mathrm{k}}{ }^{+}$and nucleophilic $\mathrm{f}_{\mathrm{k}}{ }^{-}$condensed Fukui functions over the specified carbon atoms of the imine $\mathbf{4 a}$ and imidoyl azolium intermediate $\mathbf{A}$ calculated at M06$2 \mathrm{X}$ method in conjunction with $6-311++\mathrm{G}(\mathrm{d}, \mathrm{p})$ basis set in solvent toluene. 
5. ${ }^{1} \mathrm{H}$ and ${ }^{13} \mathrm{C}$ NMR Spectra of 3,1-Benzoxazine Derivatives 2-(4-Nitrophenyl)-4-(trifluoromethyl)-4H-benzo $[d][1,3]$ oxazine $3 a$
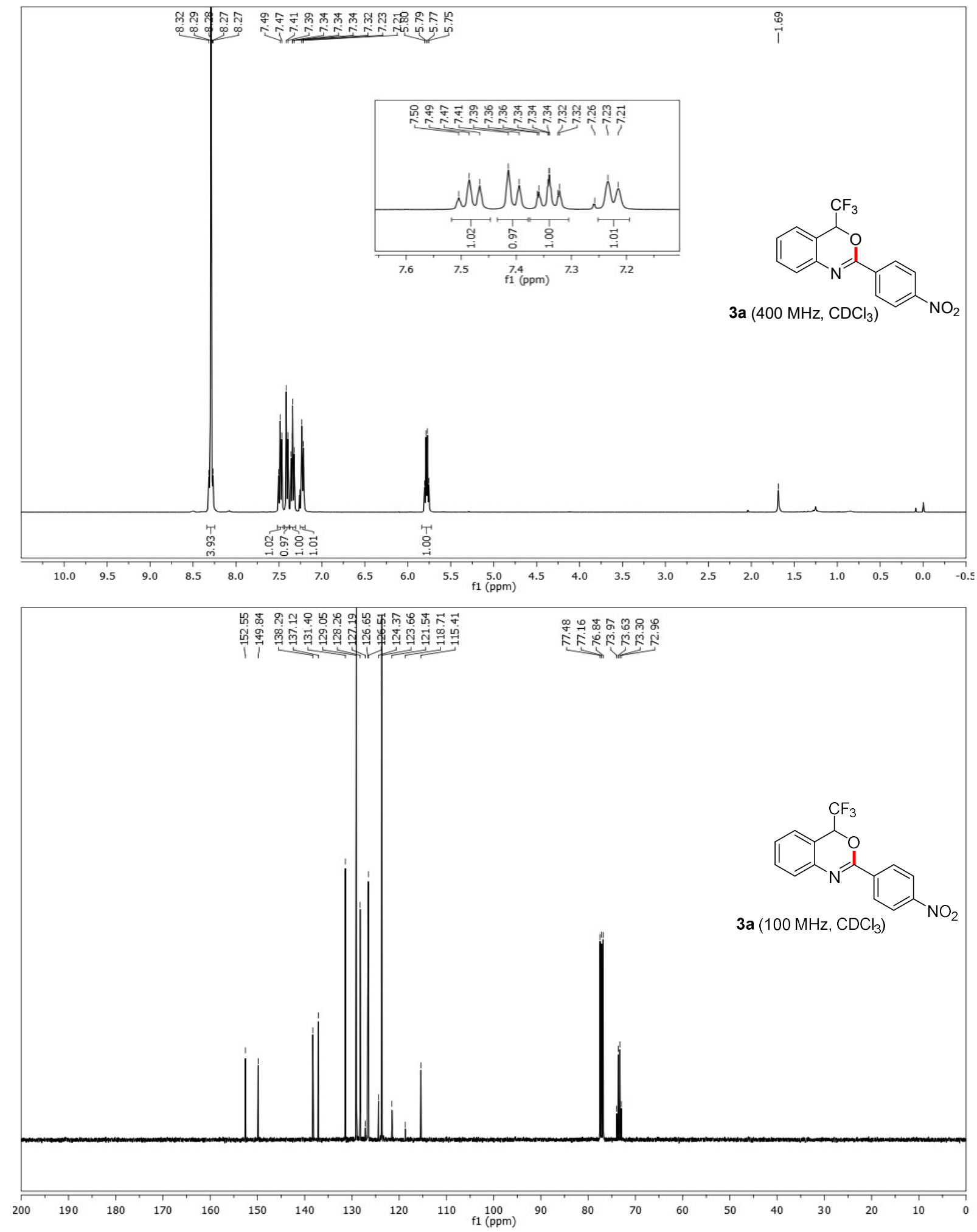

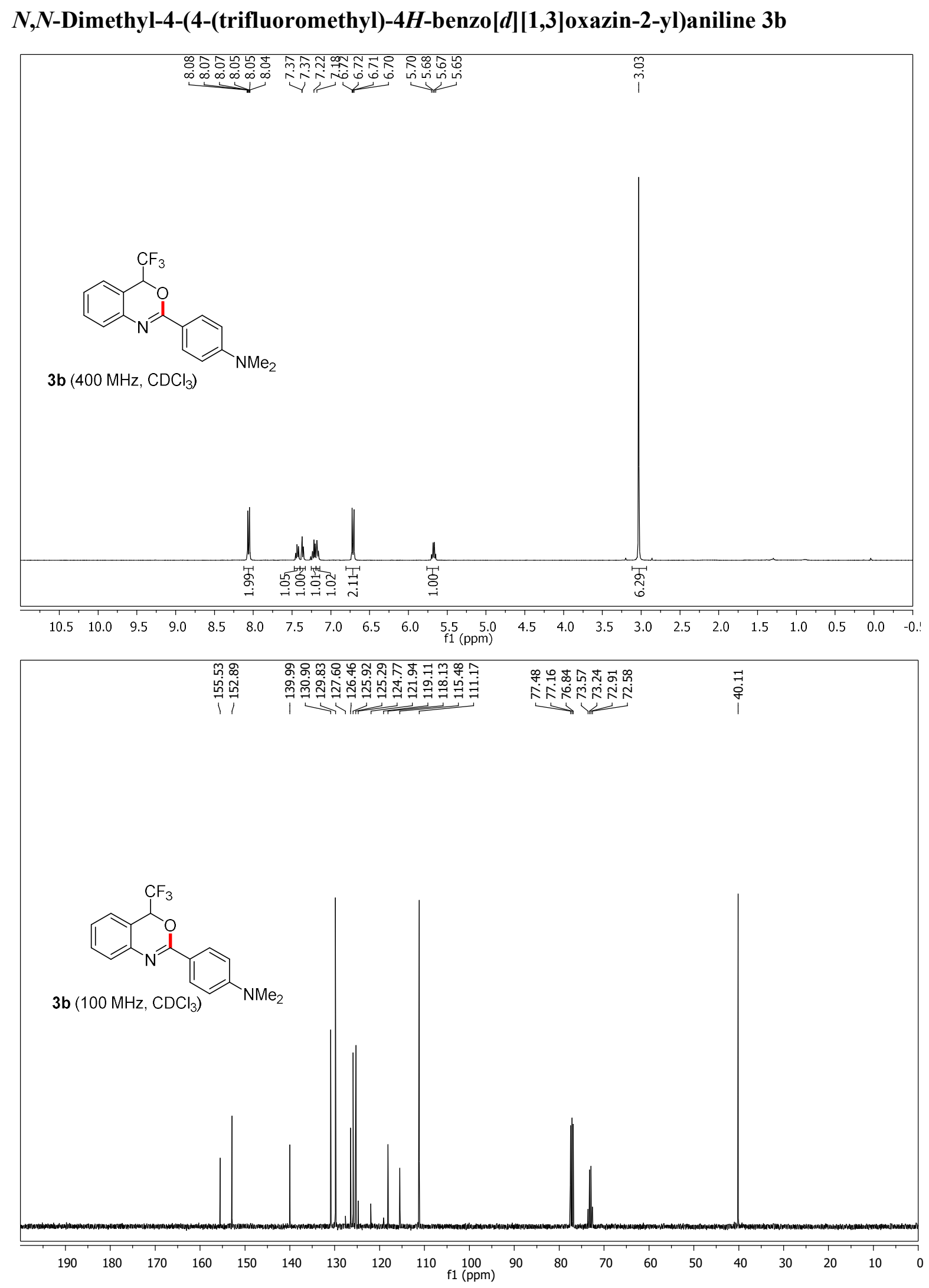

S16 
2-(4-Methoxyphenyl)-4-(trifluoromethyl)-4H-benzo[d][1,3]oxazine 3c
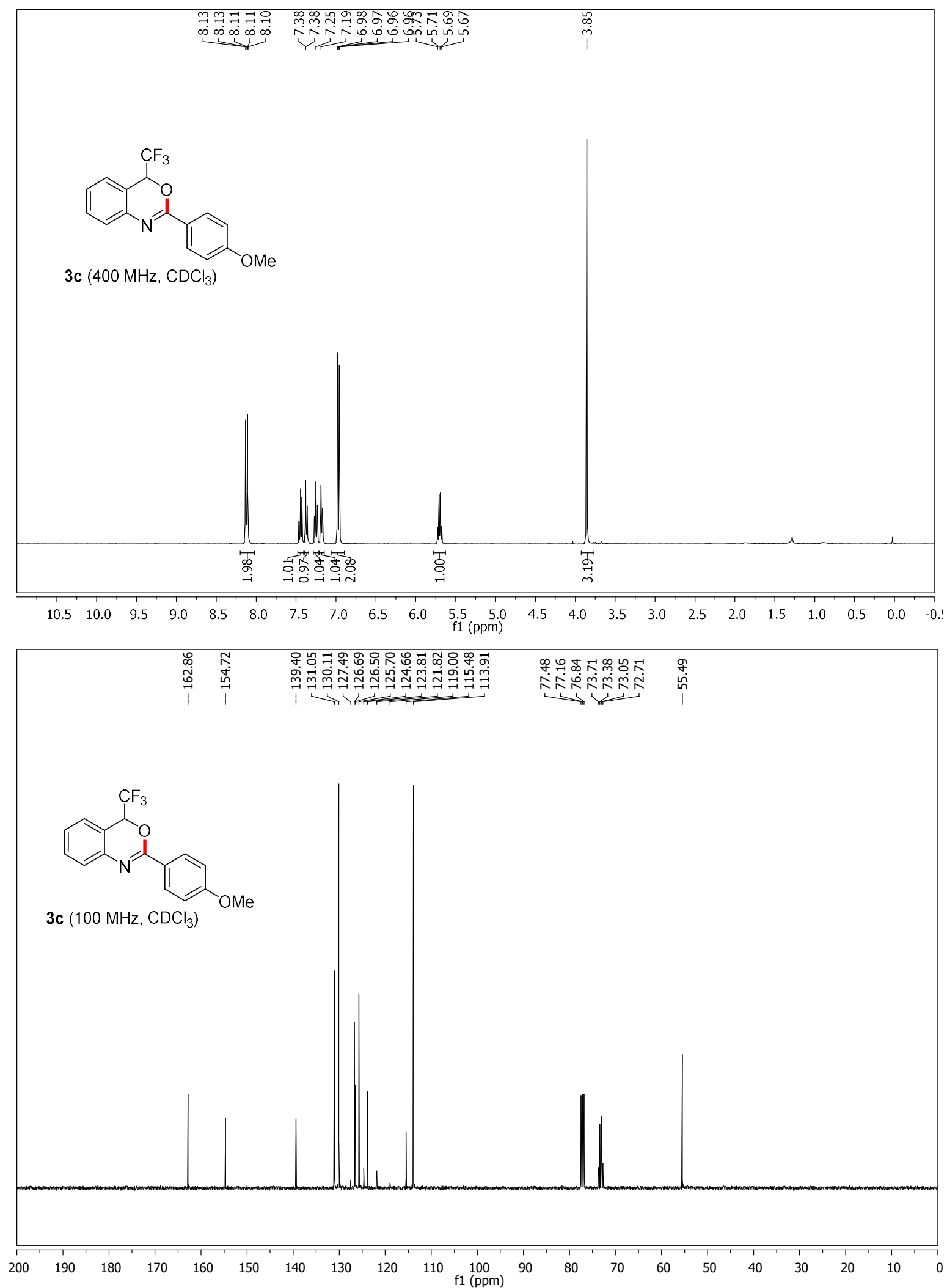


\section{2-(4-Bromophenyl)-4-(trifluoromethyl)-4H-benzo $[d][1,3]$ oxazine 3d}
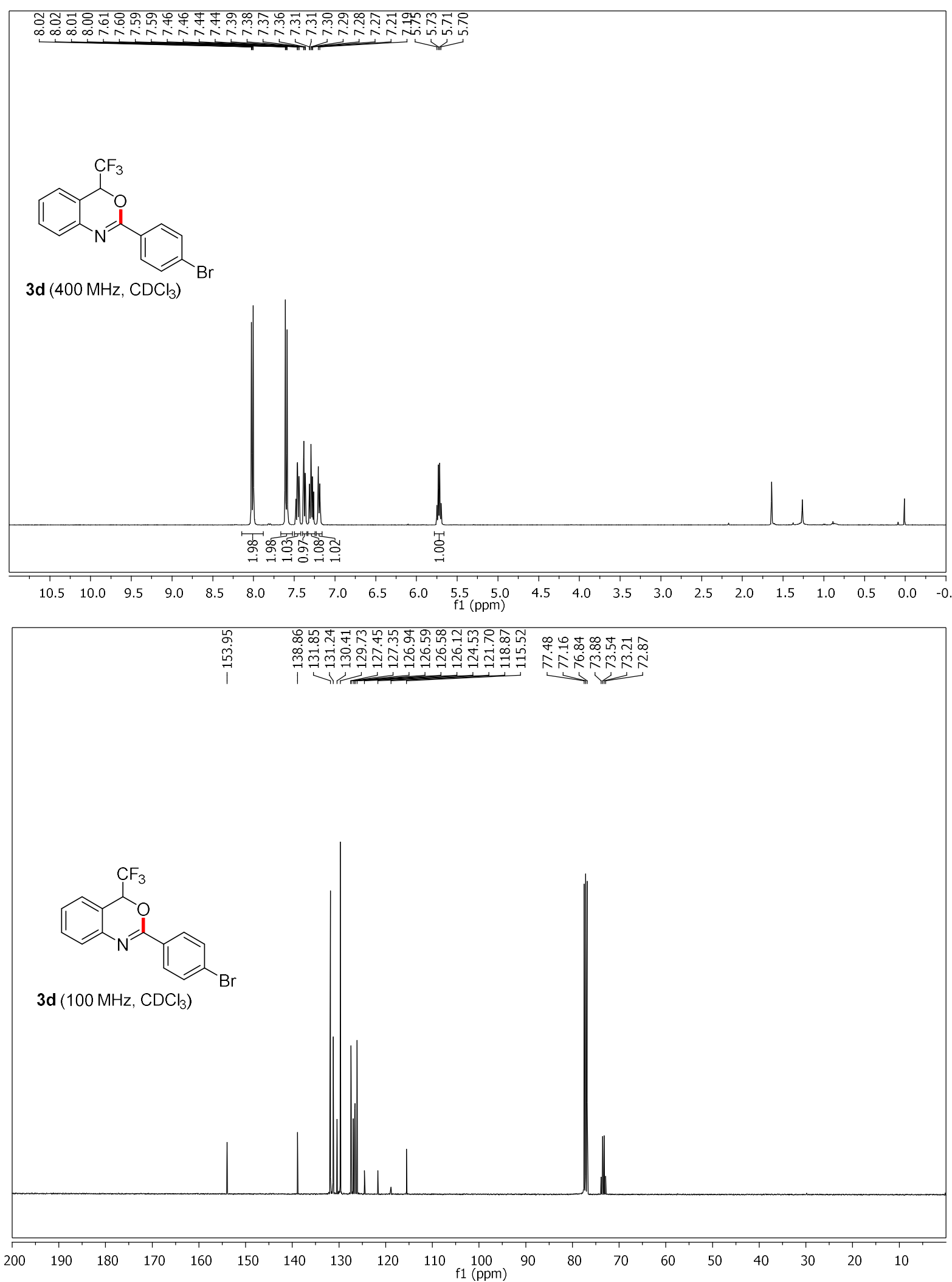


\section{2-(4-Chlorophenyl)-4-(trifluoromethyl)-4H-benzo[ $d][1,3]$ oxazine $3 e$}
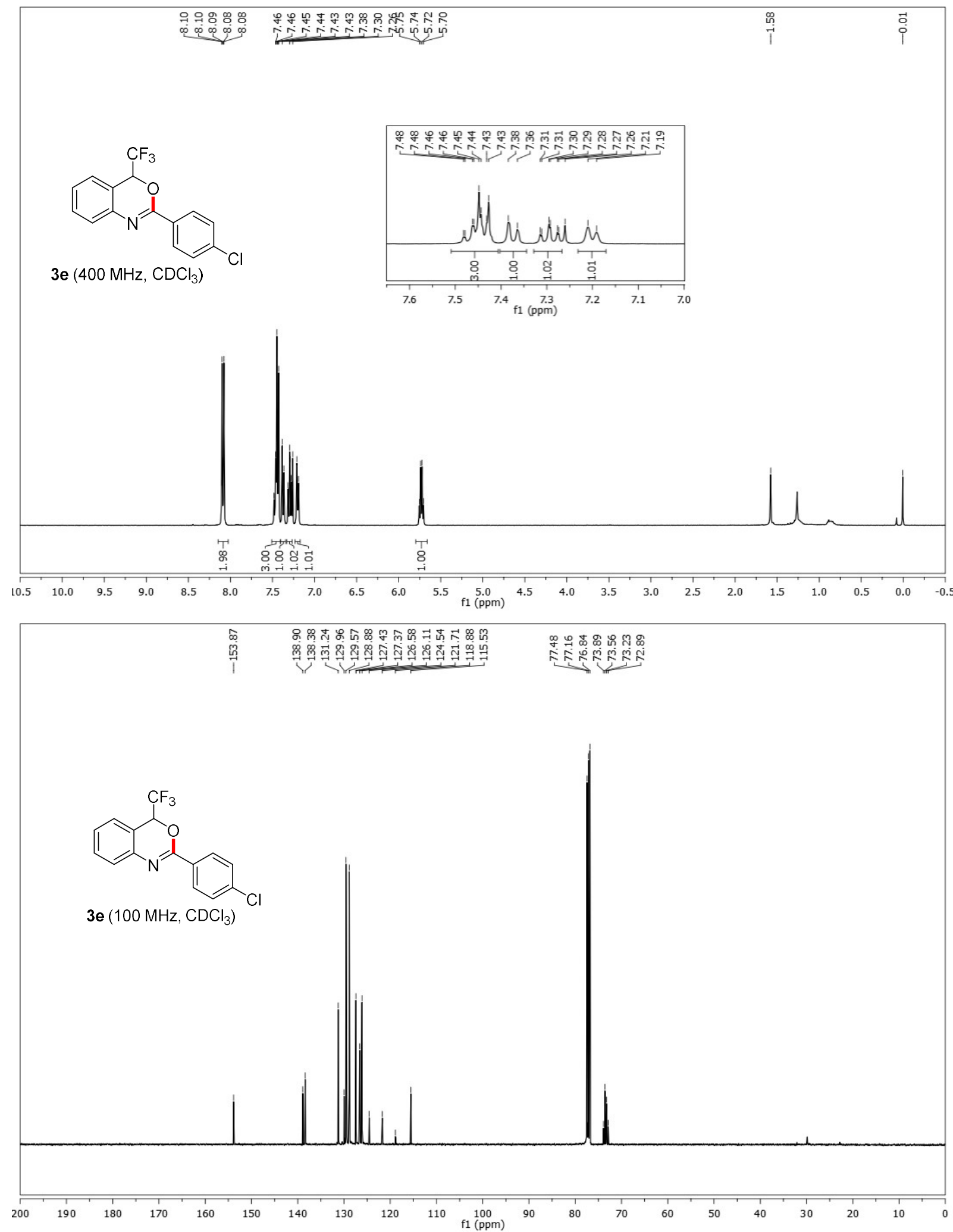


\section{4-(4-(Trifluoromethyl)-4H-benzo $[d][1,3]$ oxazin-2-yl)benzonitrile $3 f$}
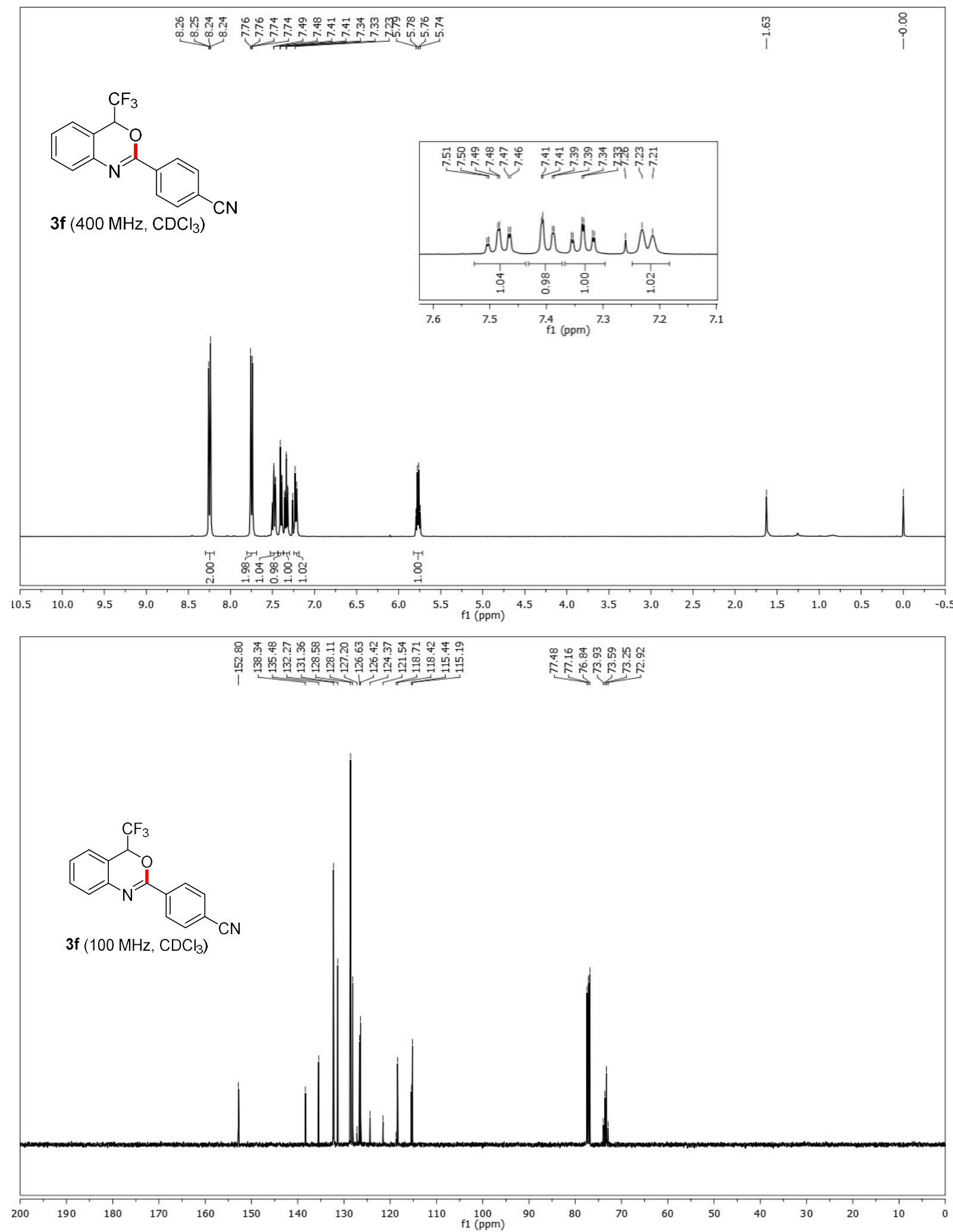
2-(3,4-Dimethoxyphenyl)-4-(trifluoromethyl)-4H-benzo $[d][1,3]$ oxazine $3 \mathrm{~g}$
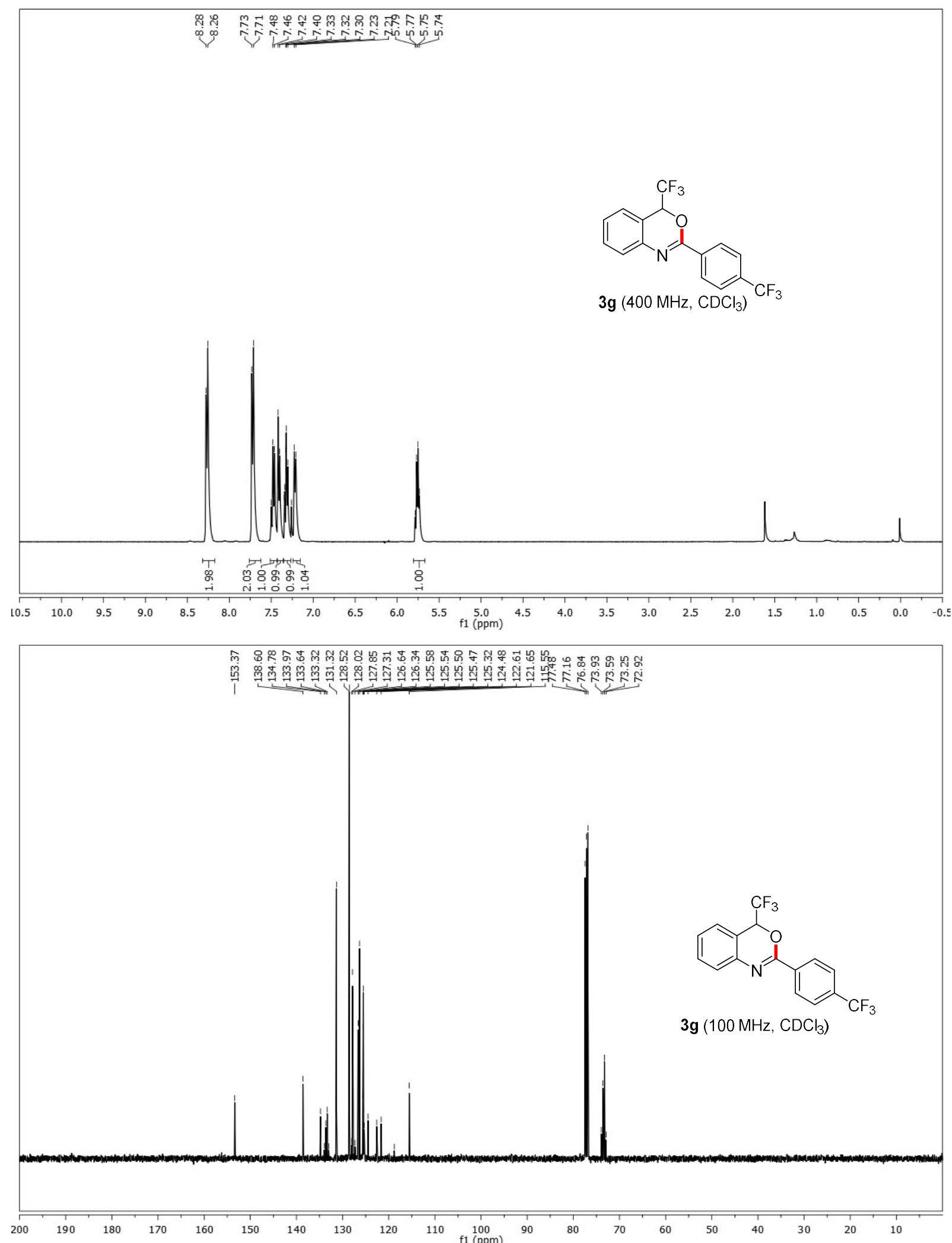
2-(3-Methoxyphenyl)-4-(trifluoromethyl)-4H-benzo[d][1,3]oxazine $3 \mathrm{~h}$
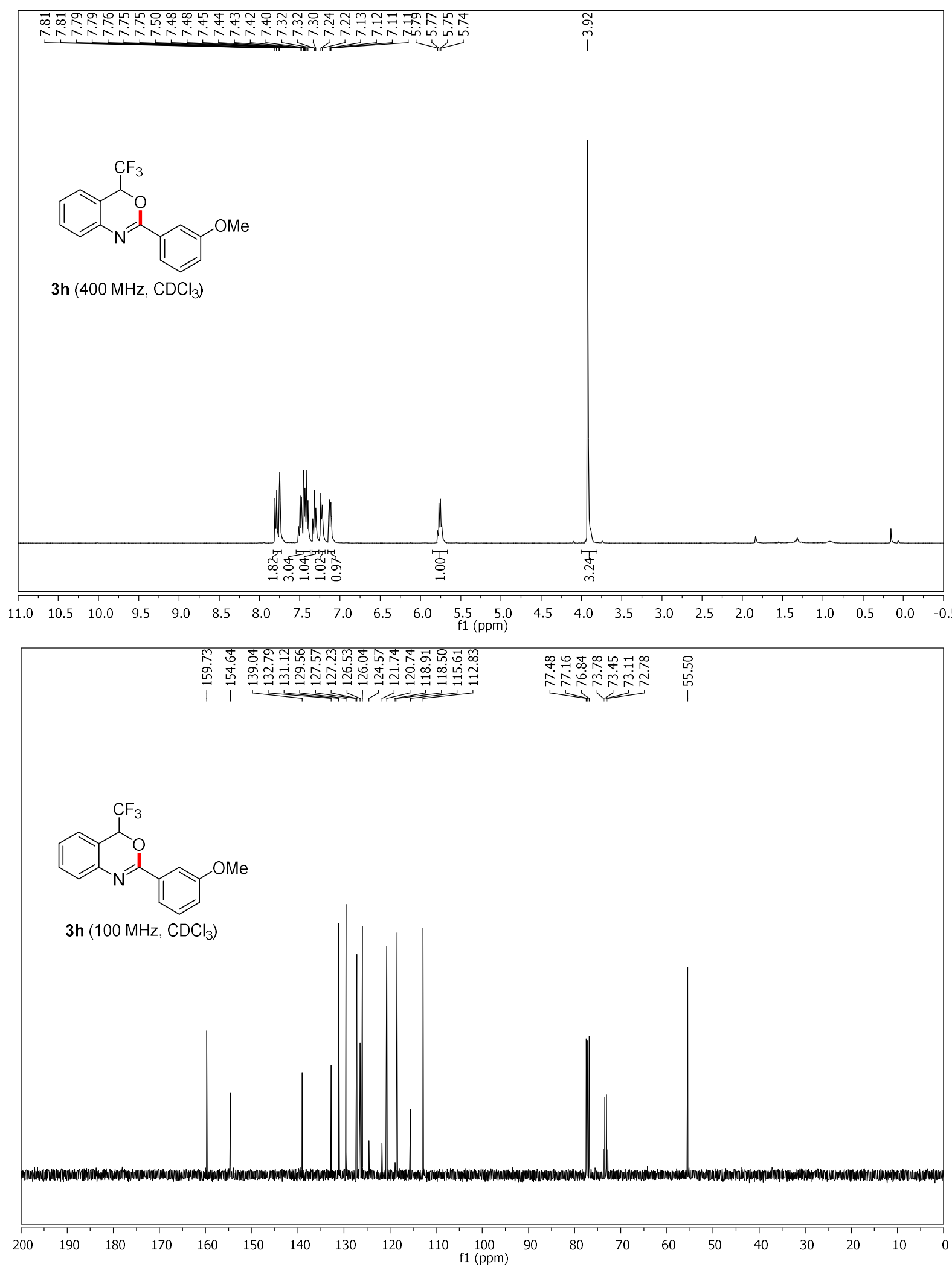
2-(3-Nitrophenyl)-4-(trifluoromethyl)-4H-benzo $[d][1,3]$ oxazine $3 i$
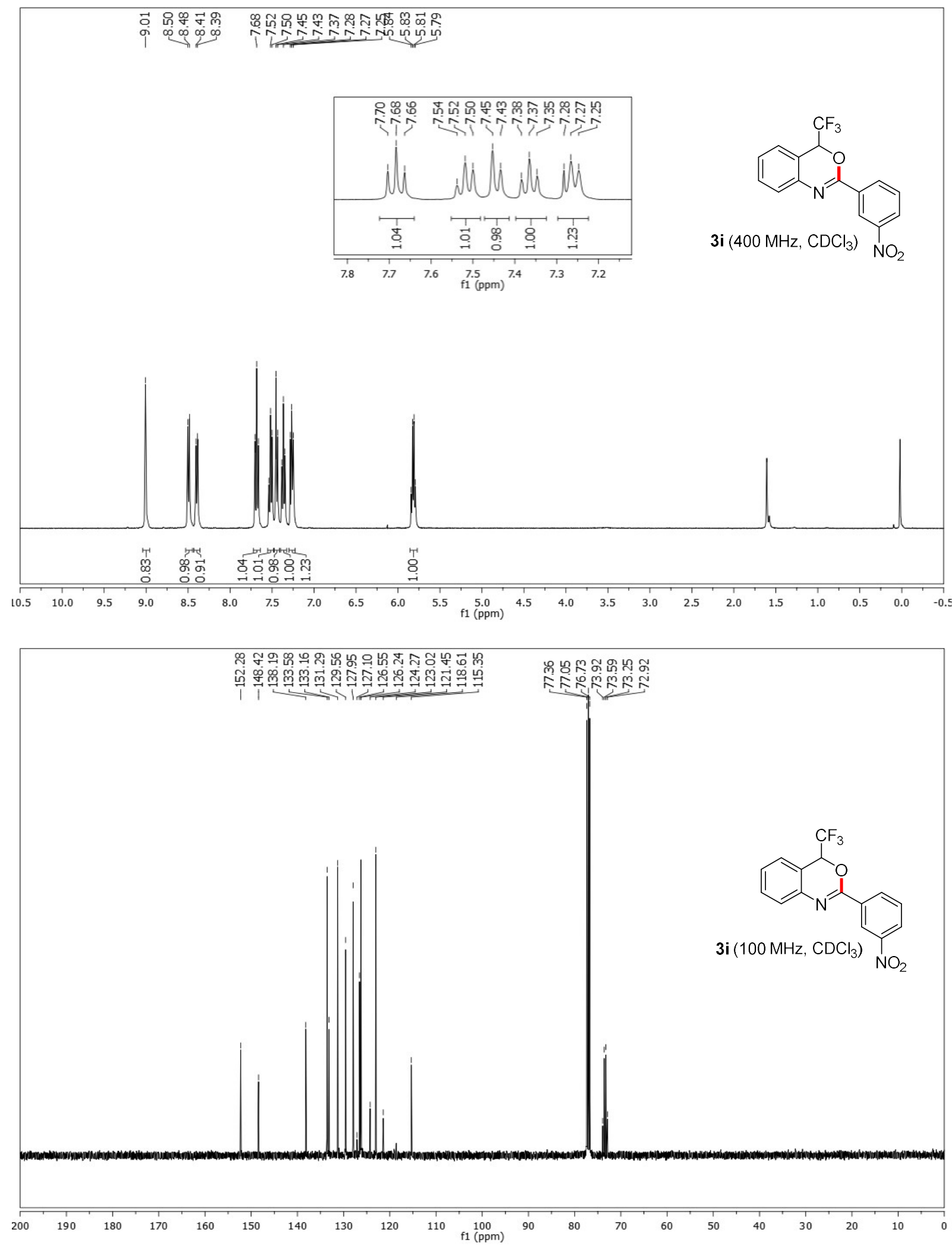


\section{2-(2-Nitrophenyl)-4-(trifluoromethyl)-4H-benzo[d] [1,3]oxazine $3 \mathrm{j}$}
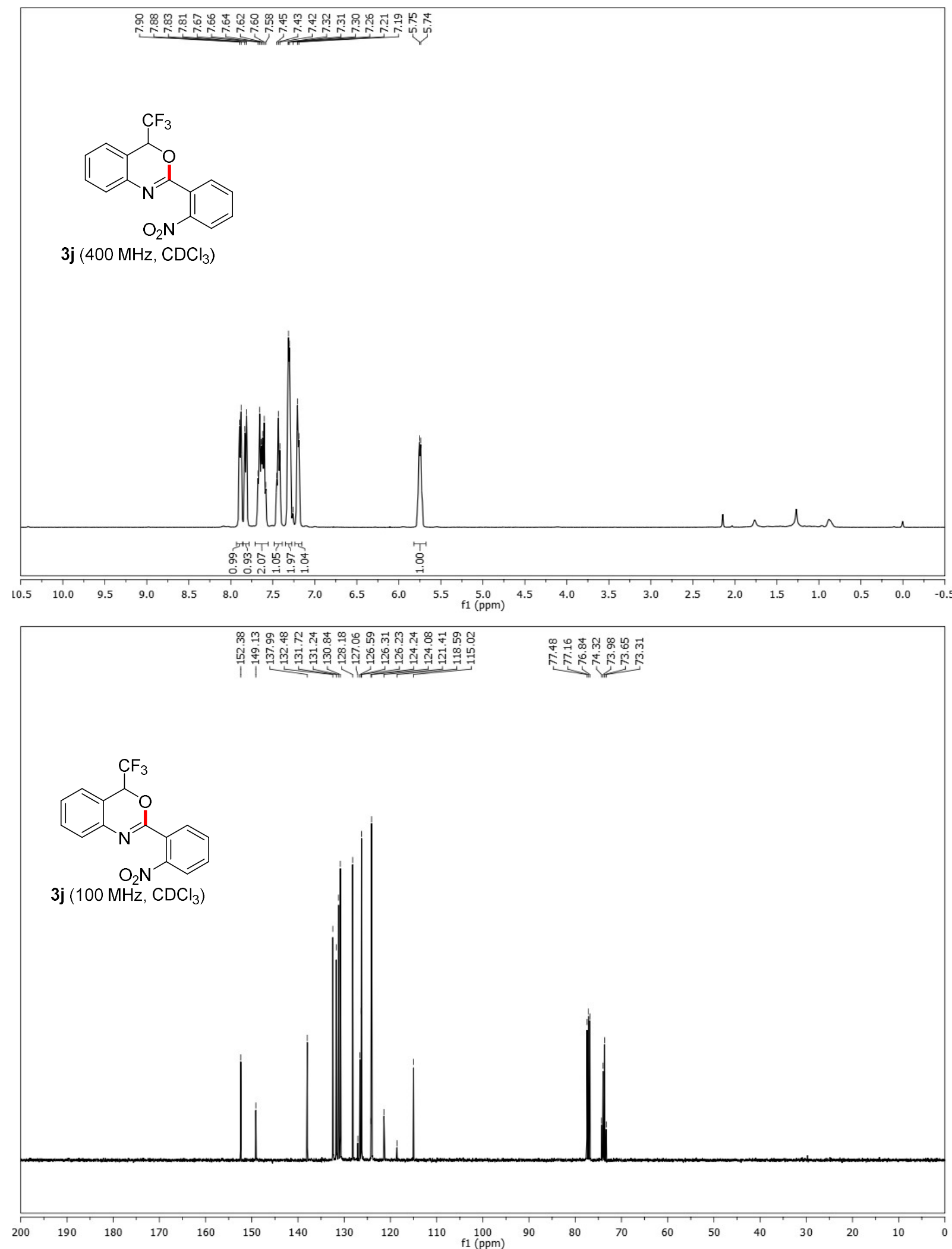


\section{2-(3,4-Dimethoxyphenyl)-4-(trifluoromethyl)-4H-benzo $[d][1,3]$ oxazine $3 \mathrm{k}$}
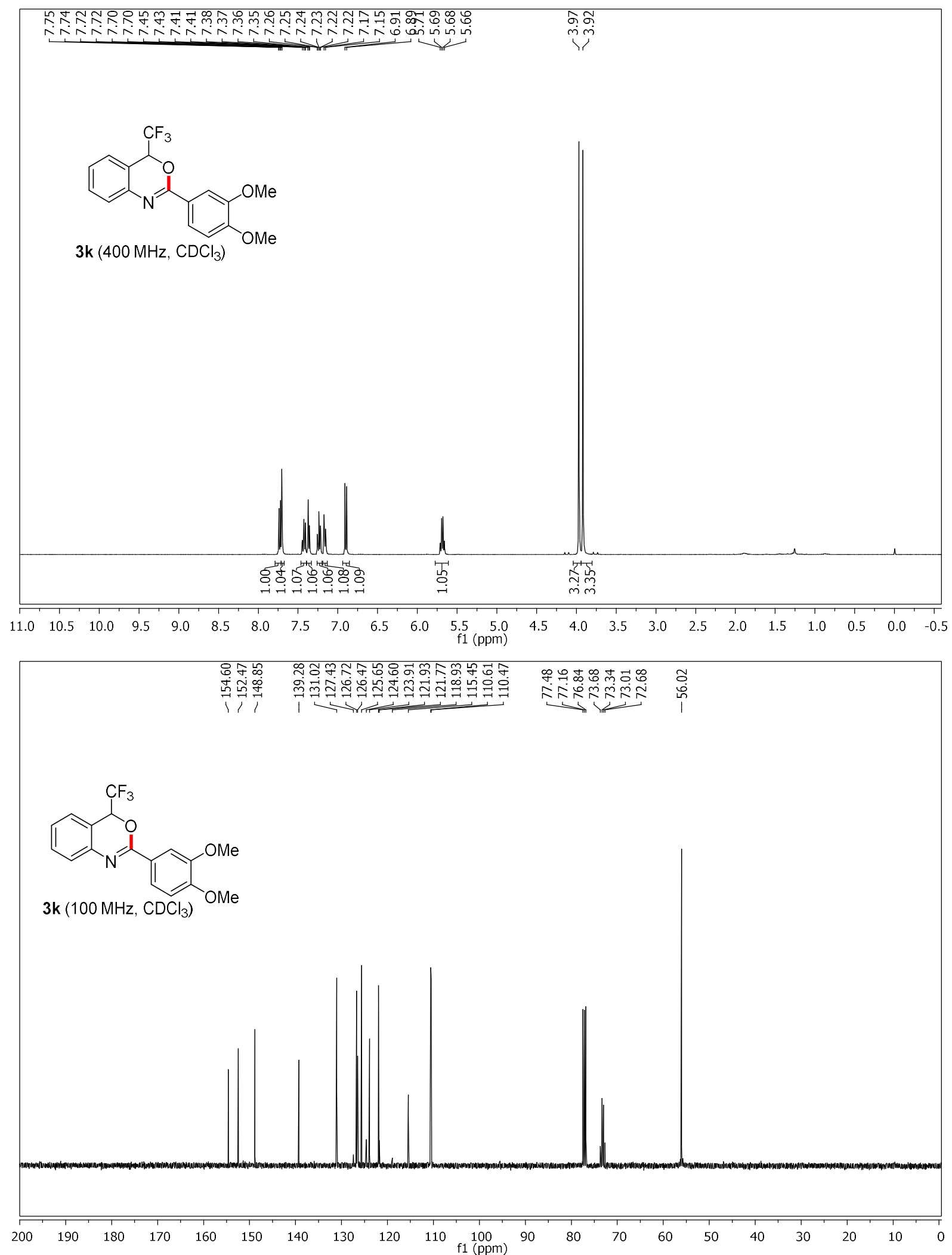
2-(2-Bromo-4-methoxyphenyl)-4-(trifluoromethyl)-4H-benzo[d][1,3]oxazine 3 I
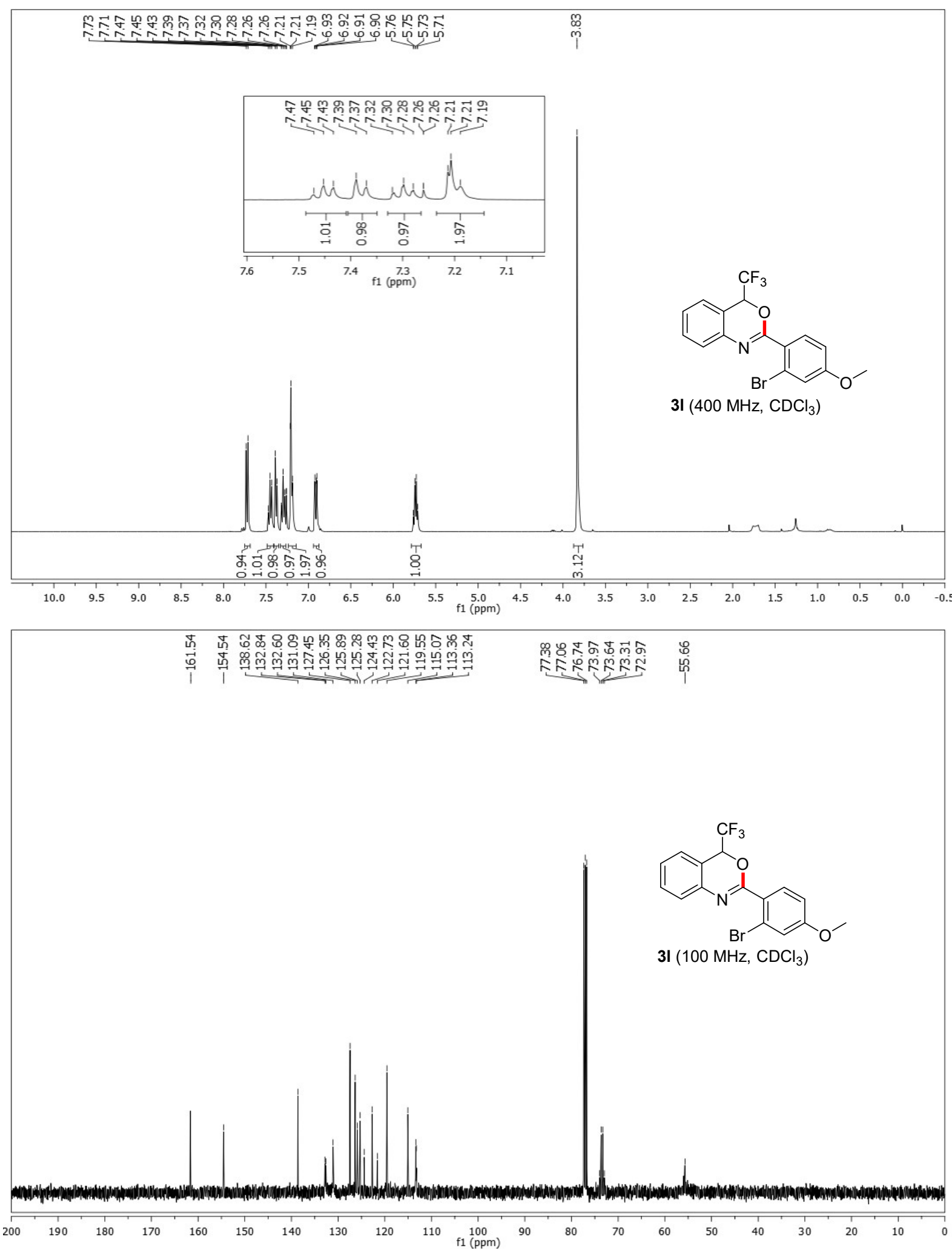
2-Methoxy-6-(4-(trifluoromethyl)-4H-benzo[d][1,3]oxazin-2-yl)phenol 3m

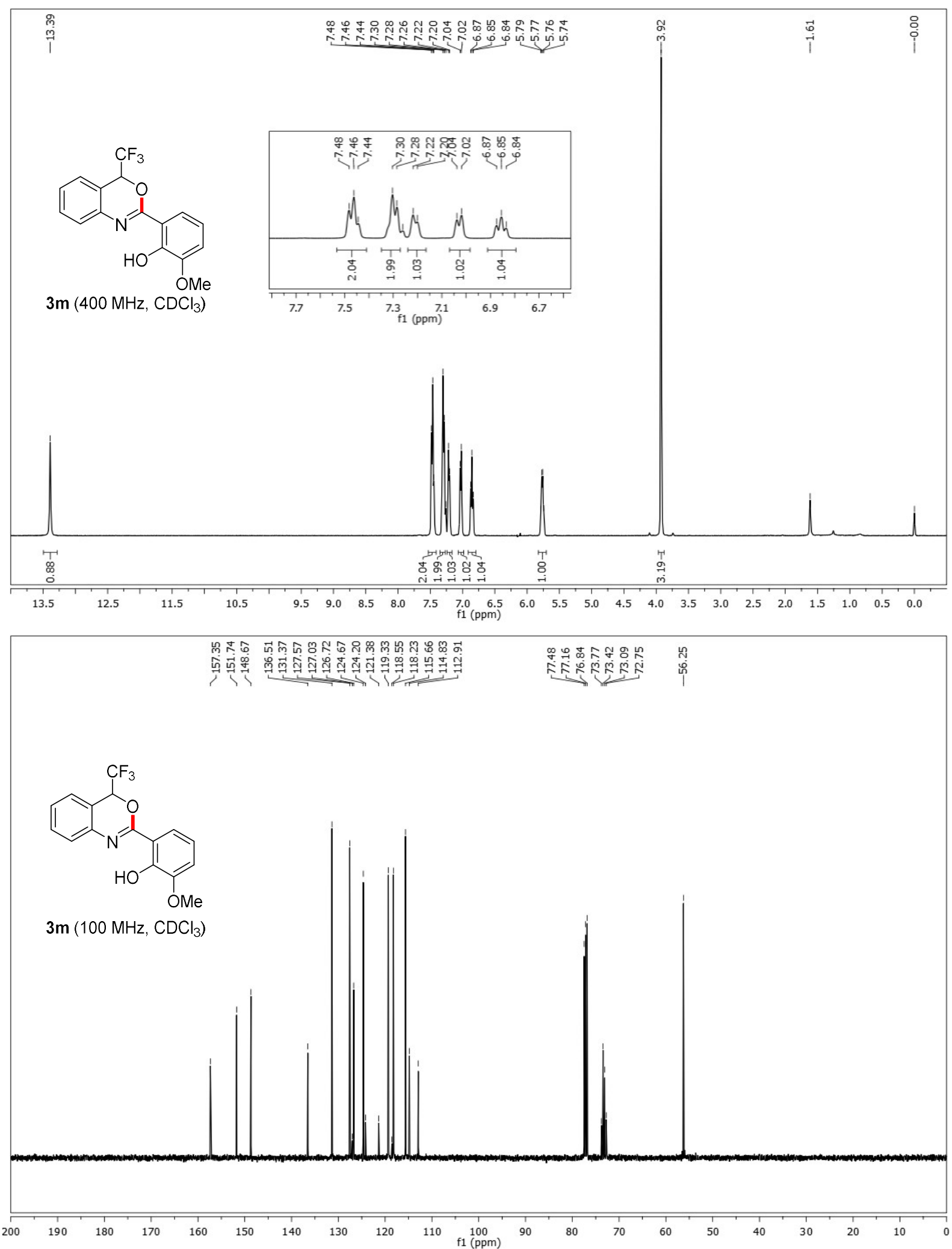


2-(Naphthalen-2-yl)-4-(trifluoromethyl)-4H-benzo[d][1,3]oxazine 3n
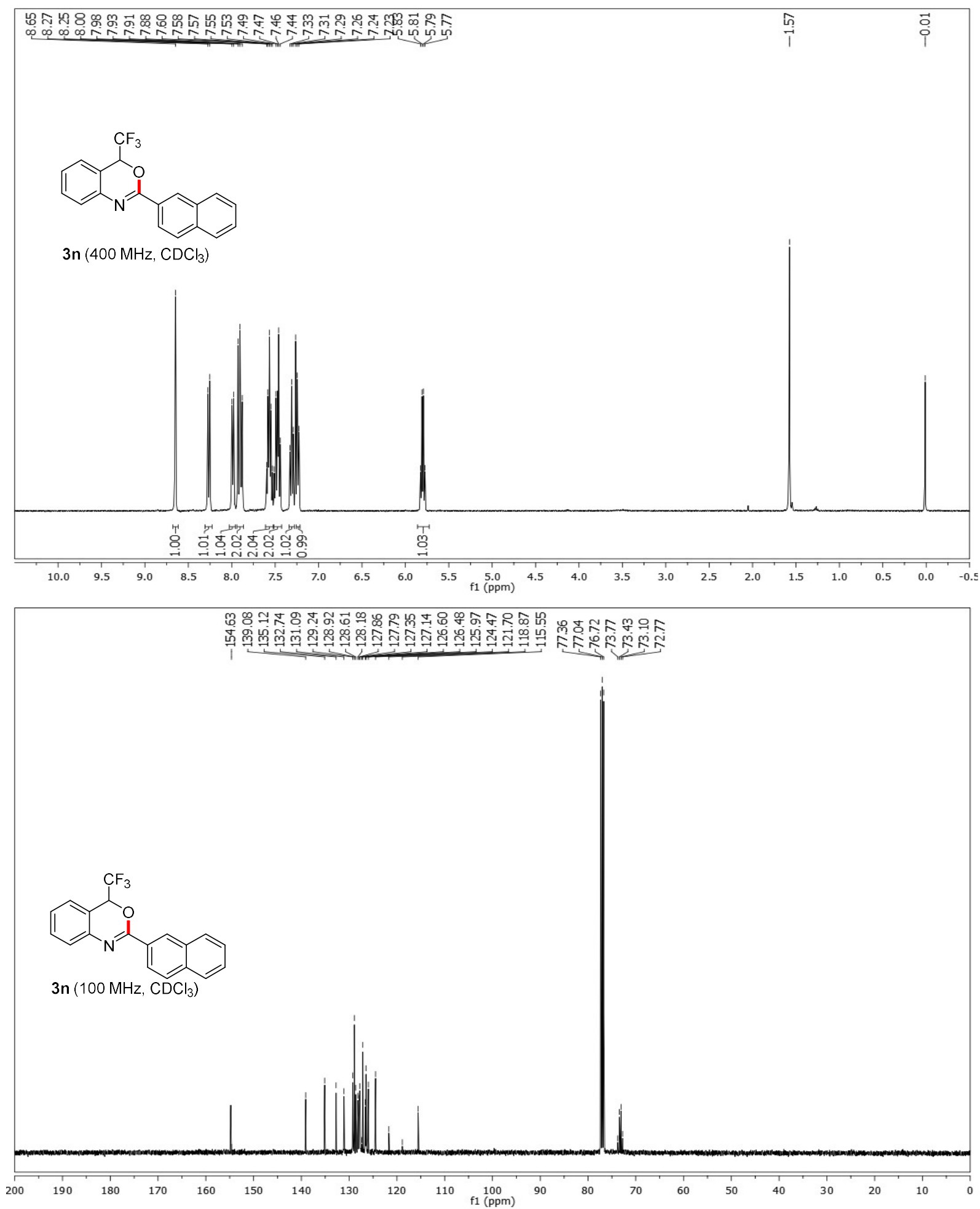
2-(Pyren-1-yl)-4-(trifluoromethyl)-4H-benzo[d][1,3]oxazine 3o
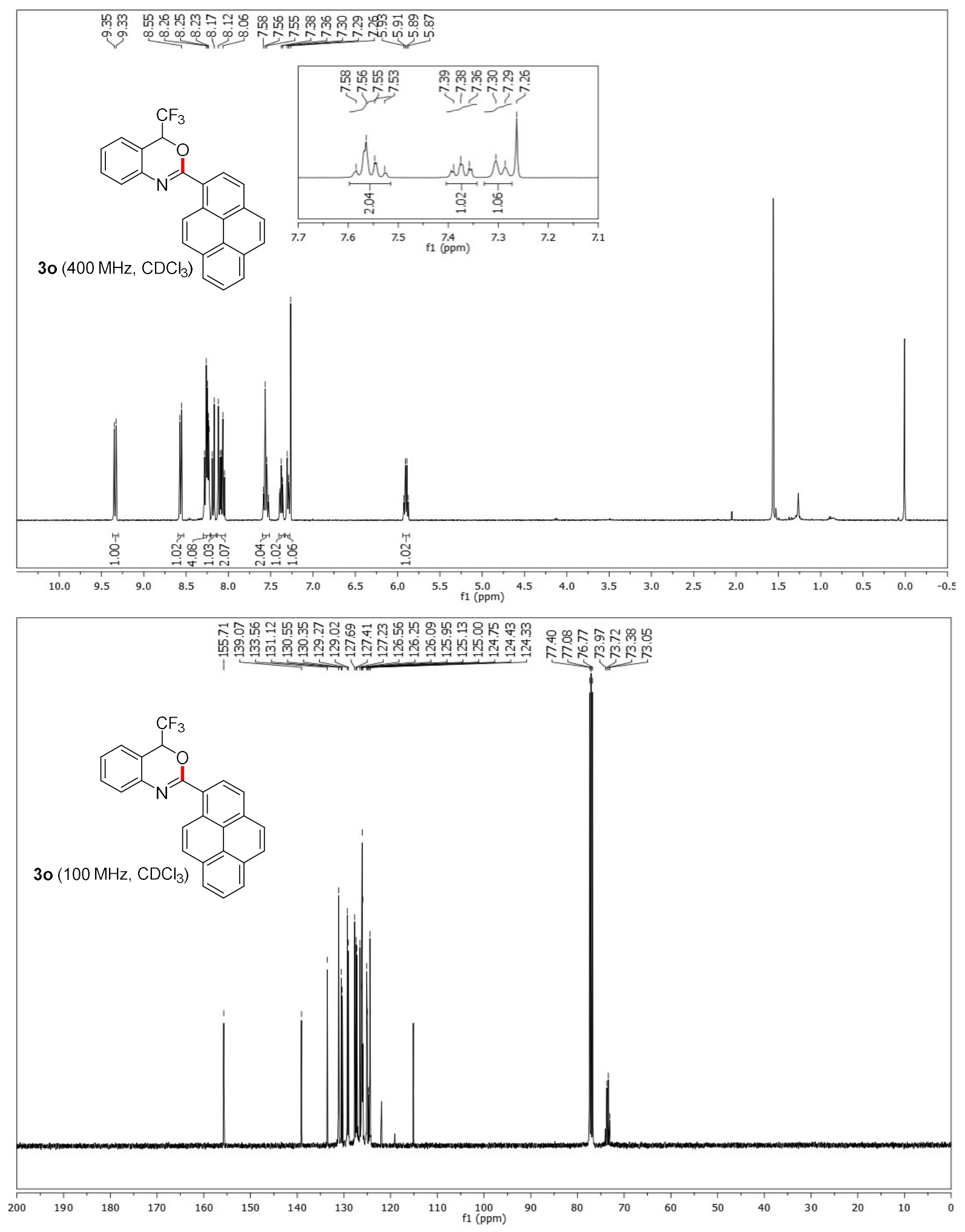

S29 
(E)-2-(4-Nitrostyryl)-4-(trifluoromethyl)-4H-benzo[d][1,3]oxazine 3p
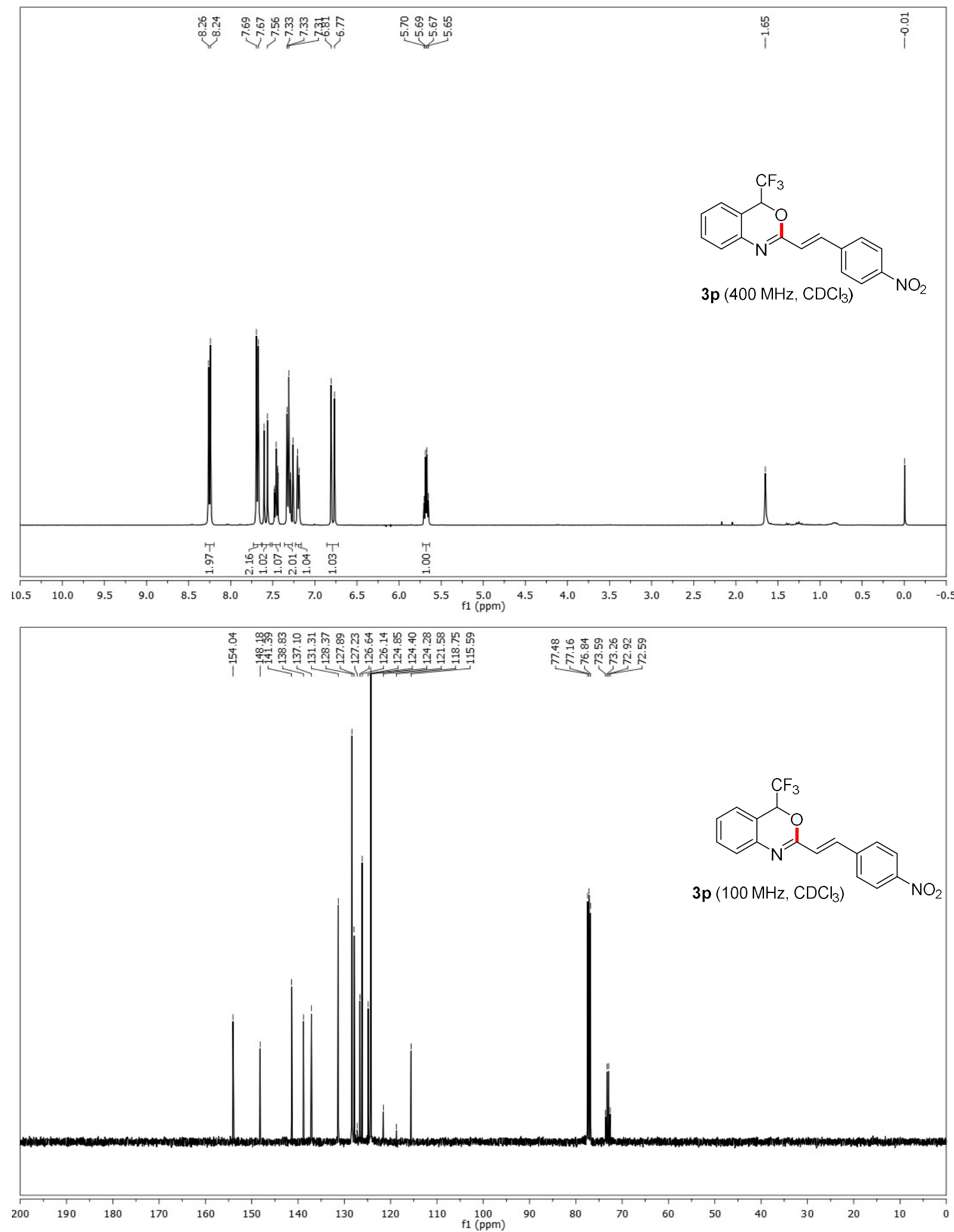
(E)-N,N-Dimethyl-4-(2-(4-(trifluoromethyl)-4H-benzo $[d][1,3]$ oxazin-2-yl)vinyl)aniline 3q
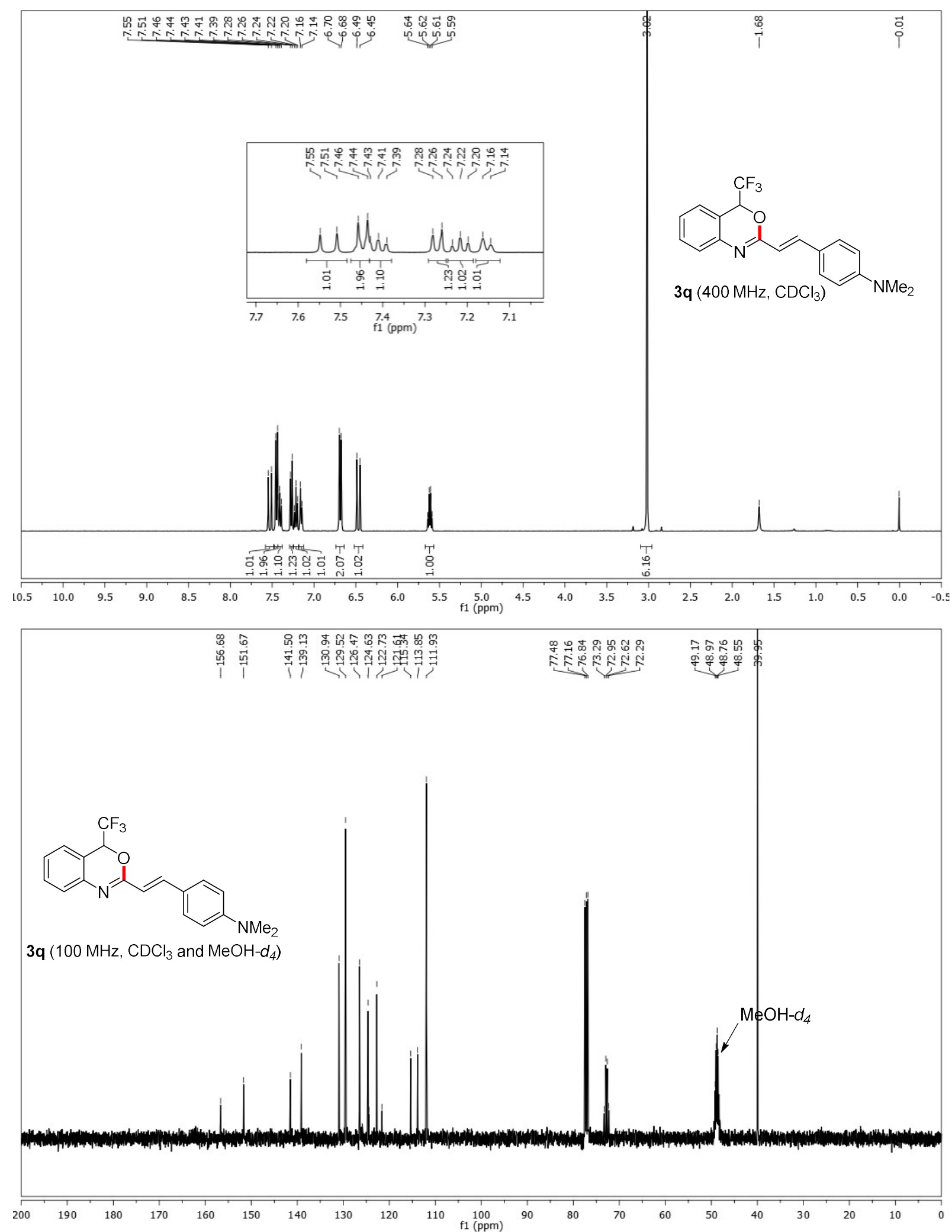


\section{2-(Pyridin-4-yl)-4-(trifluoromethyl)-4H-benzo[d][1,3]oxazine 3r}
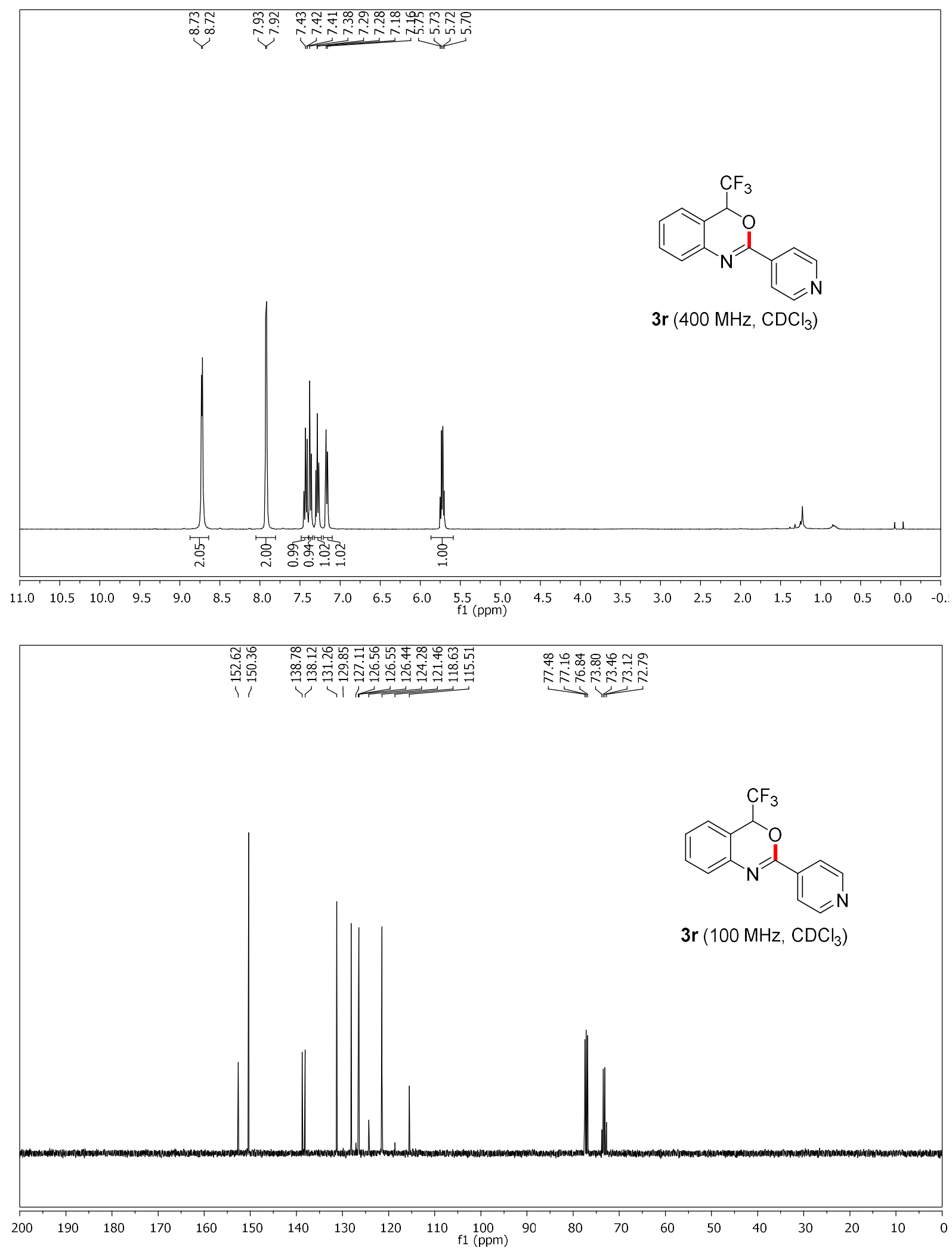
2-(Pyridin-2-yl)-4-(trifluoromethyl)-4H-benzo[d][1,3]oxazine 3s
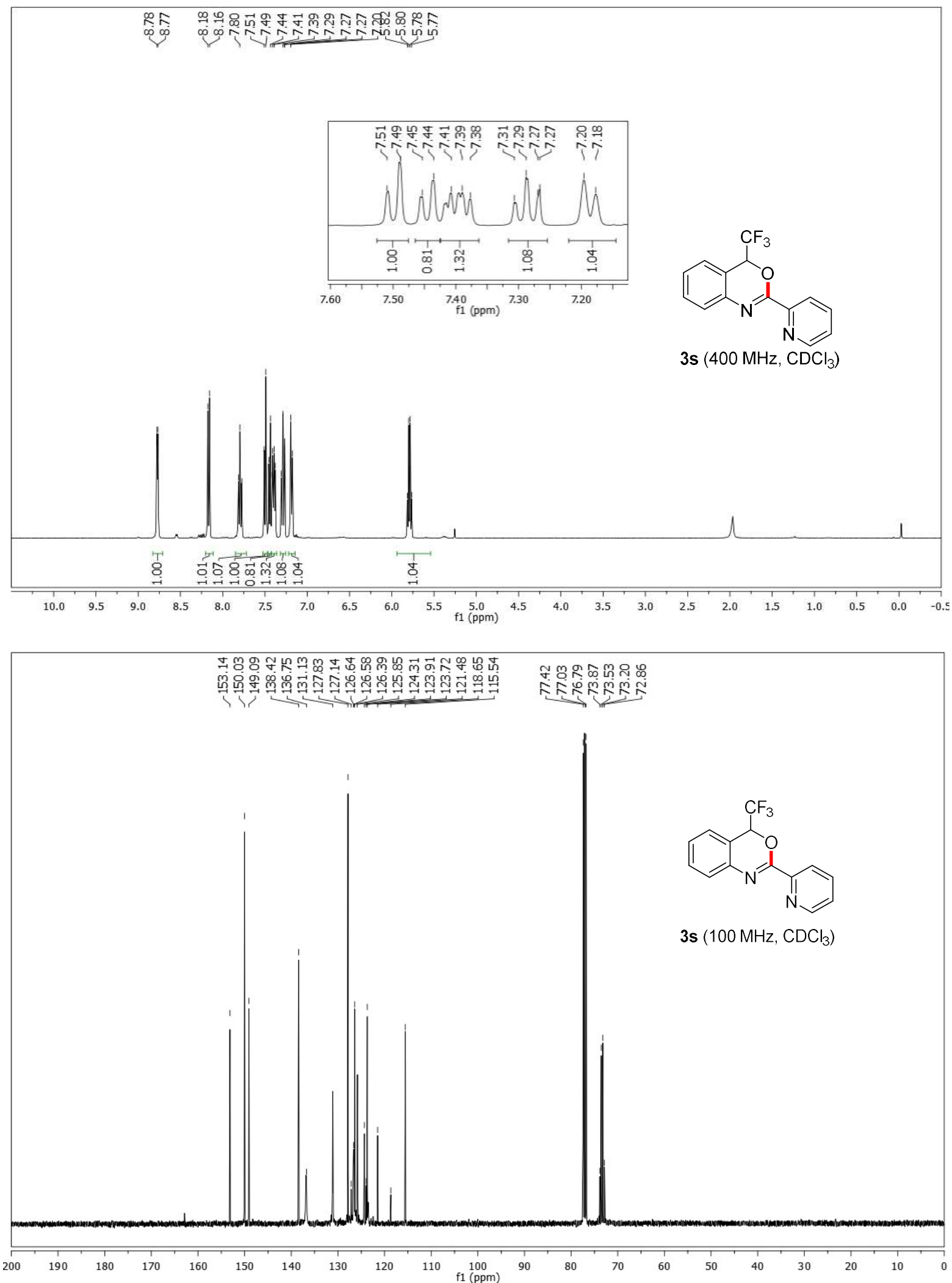
2-(Furan-2-yl)-4-(trifluoromethyl)-4H-benzo[ $[d][1,3]$ oxazine $3 t$
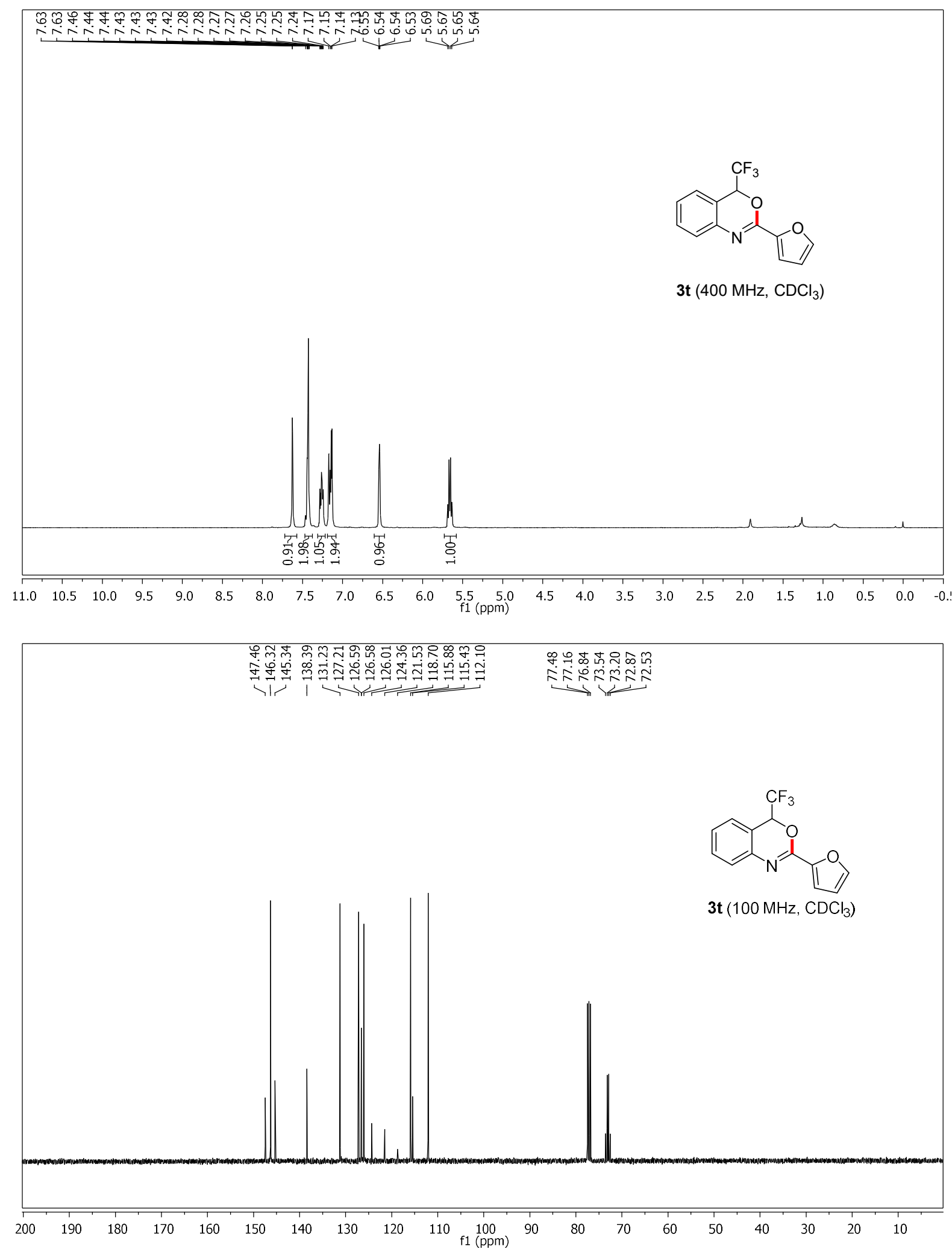
2-(Thiophen-2-yl)-4-(trifluoromethyl)-4H-benzo[d][1,3]oxazine 3u
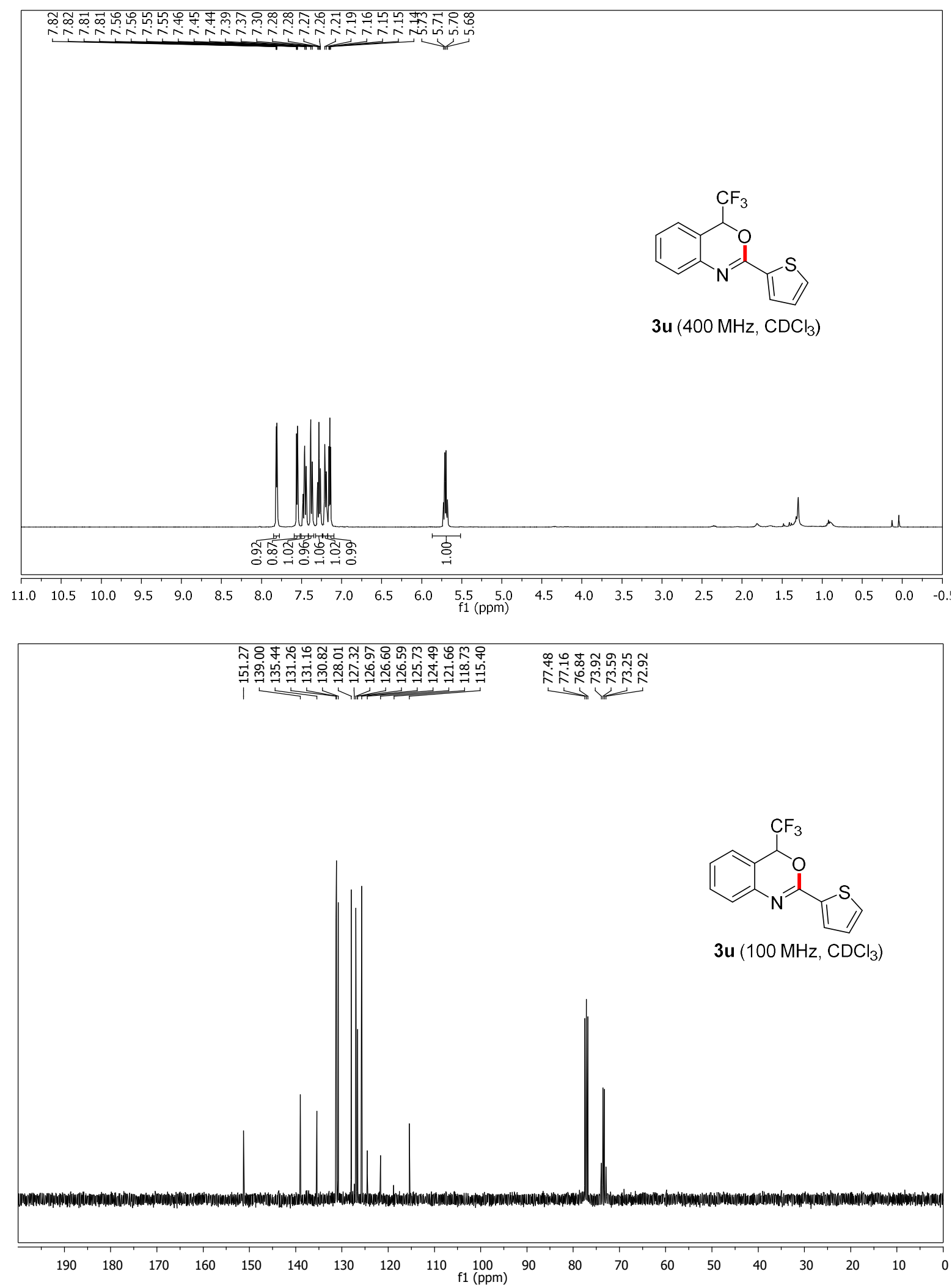
2-(1H-Pyrrol-2-yl)-4-(trifluoromethyl)-4H-benzo[d][1,3]oxazine 3v
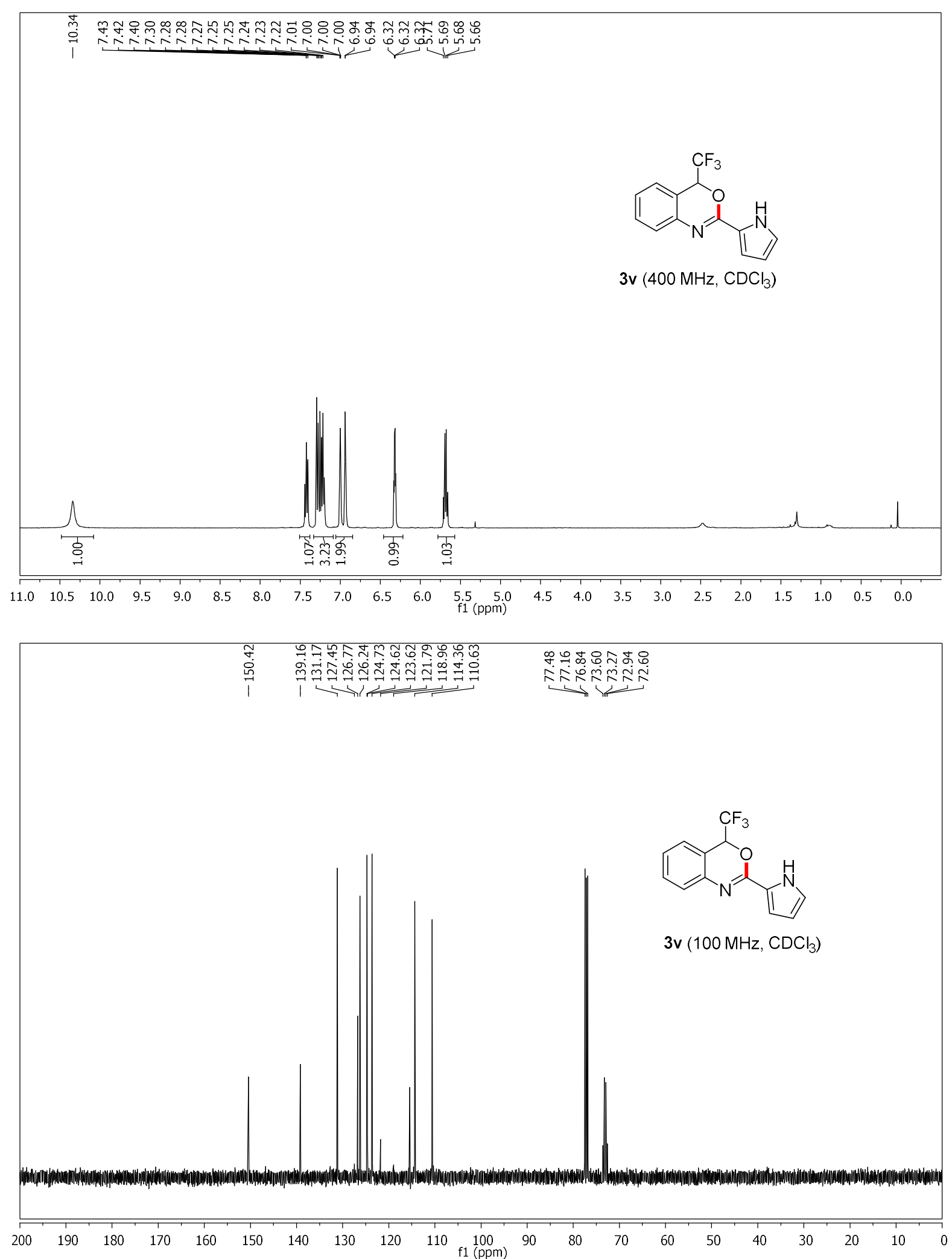
2-(Benzofuran-2-yl)-4-(trifluoromethyl)-4H-benzo[d][1,3]oxazine 3w
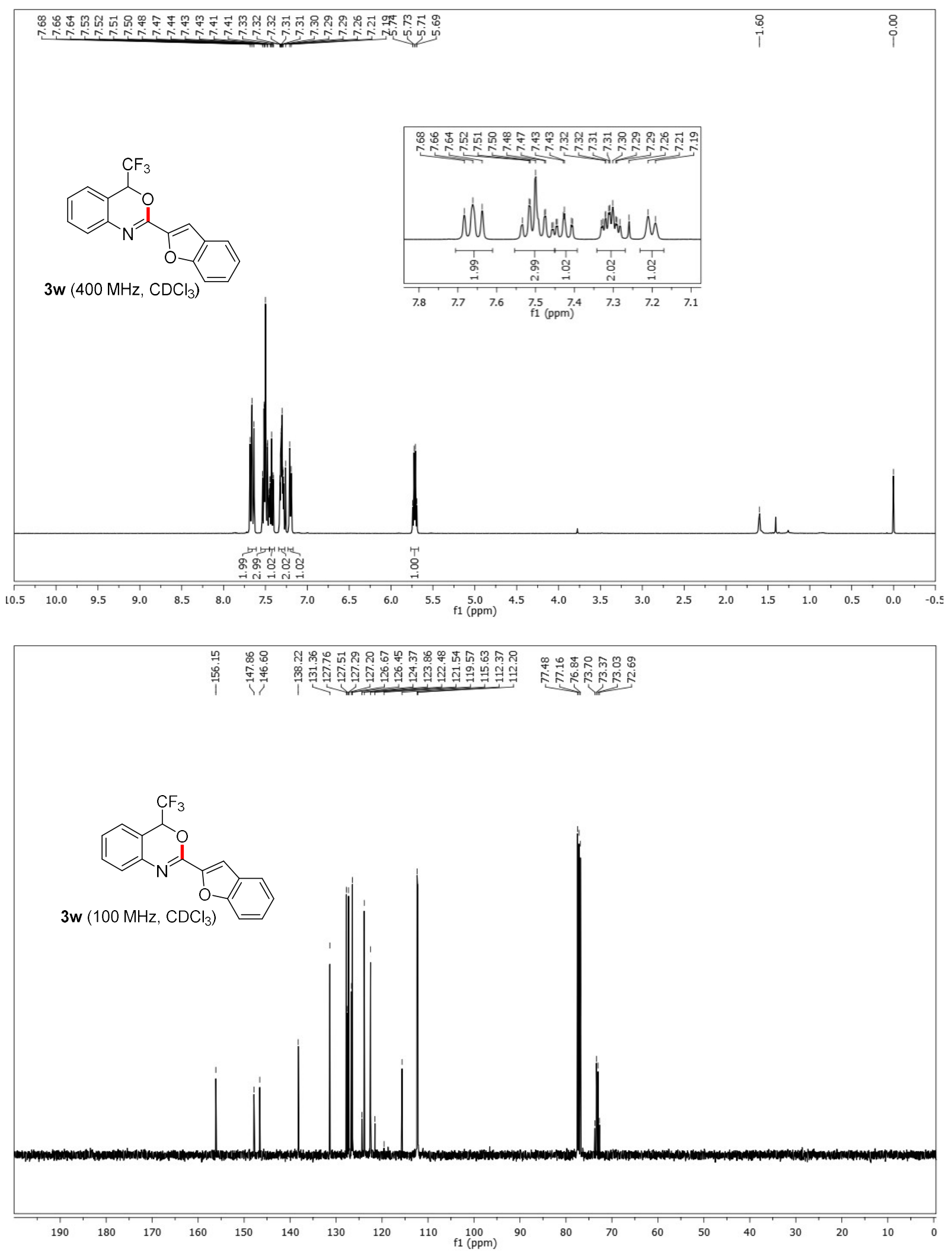

S37 
2-(Ferrocenyl)-4-(trifluoromethyl)-4H-benzo $[d][1,3]$ oxazine $3 x$
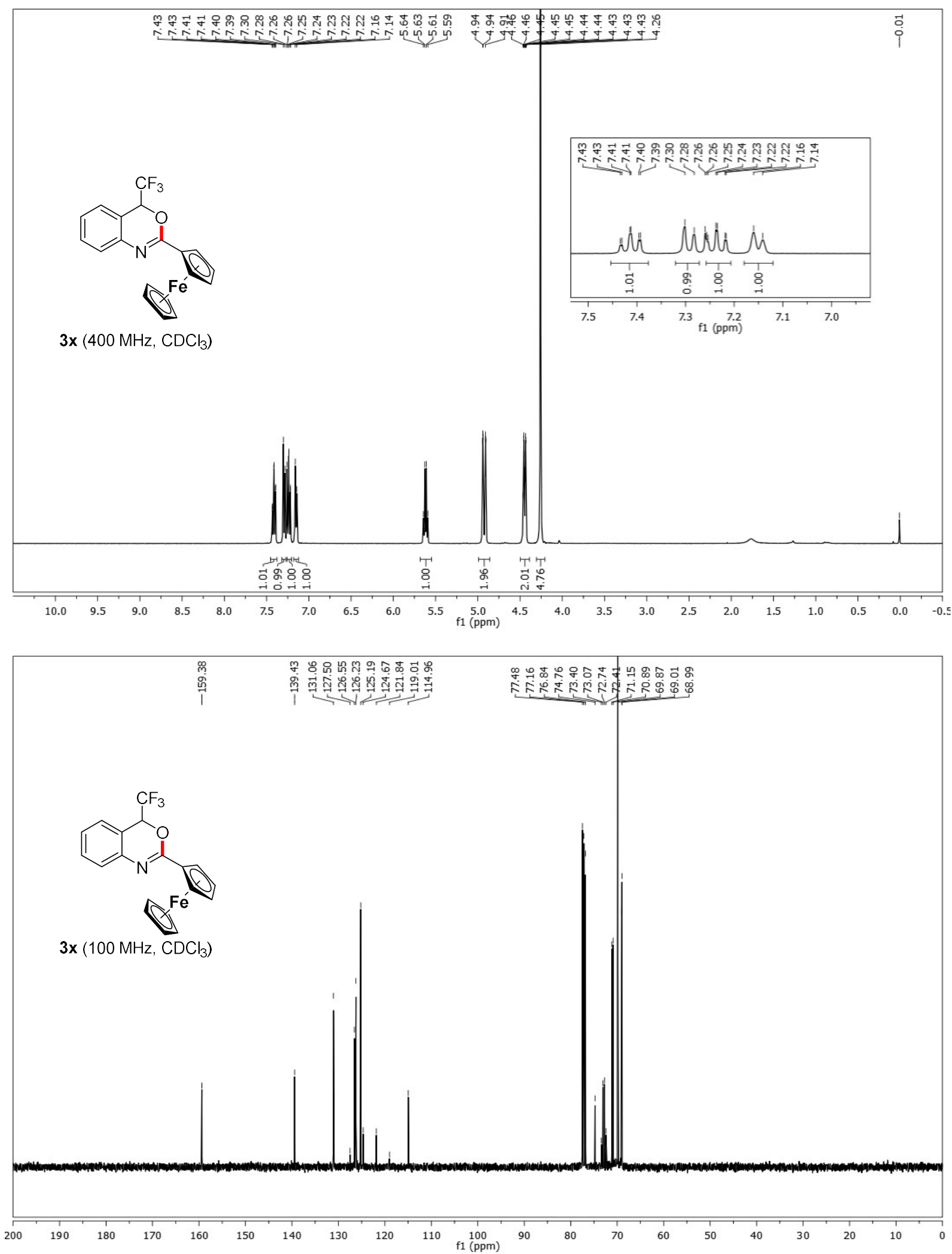
1,4-Bis(4-(trifluoromethyl)-4H-benzo[ $[d][1,3]$ oxazin-2-yl)benzene $3 y$
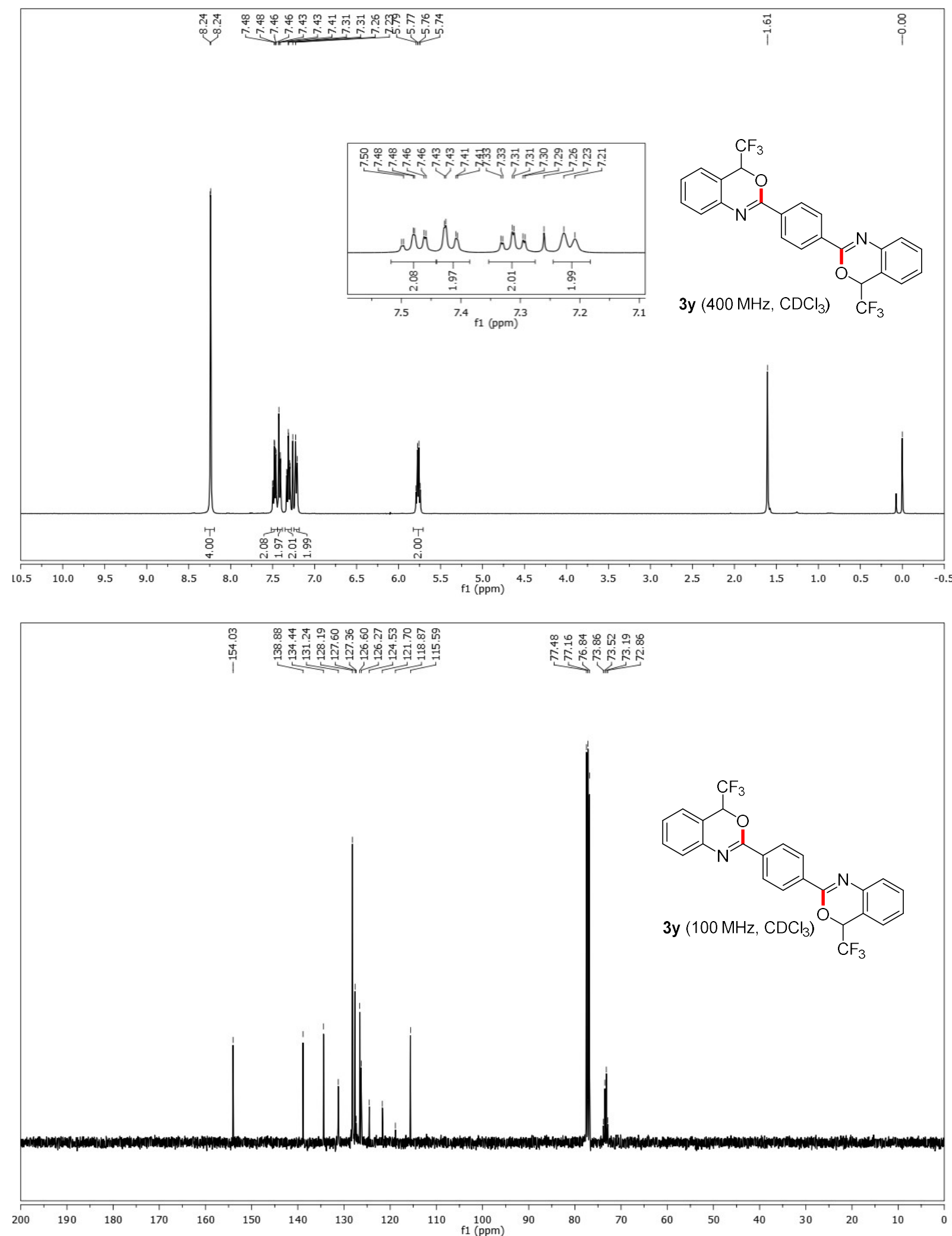
6-Methoxy-2-(4-nitrophenyl)-4-(trifluoromethyl)-4H-benzo[d][1,3]oxazine 3z
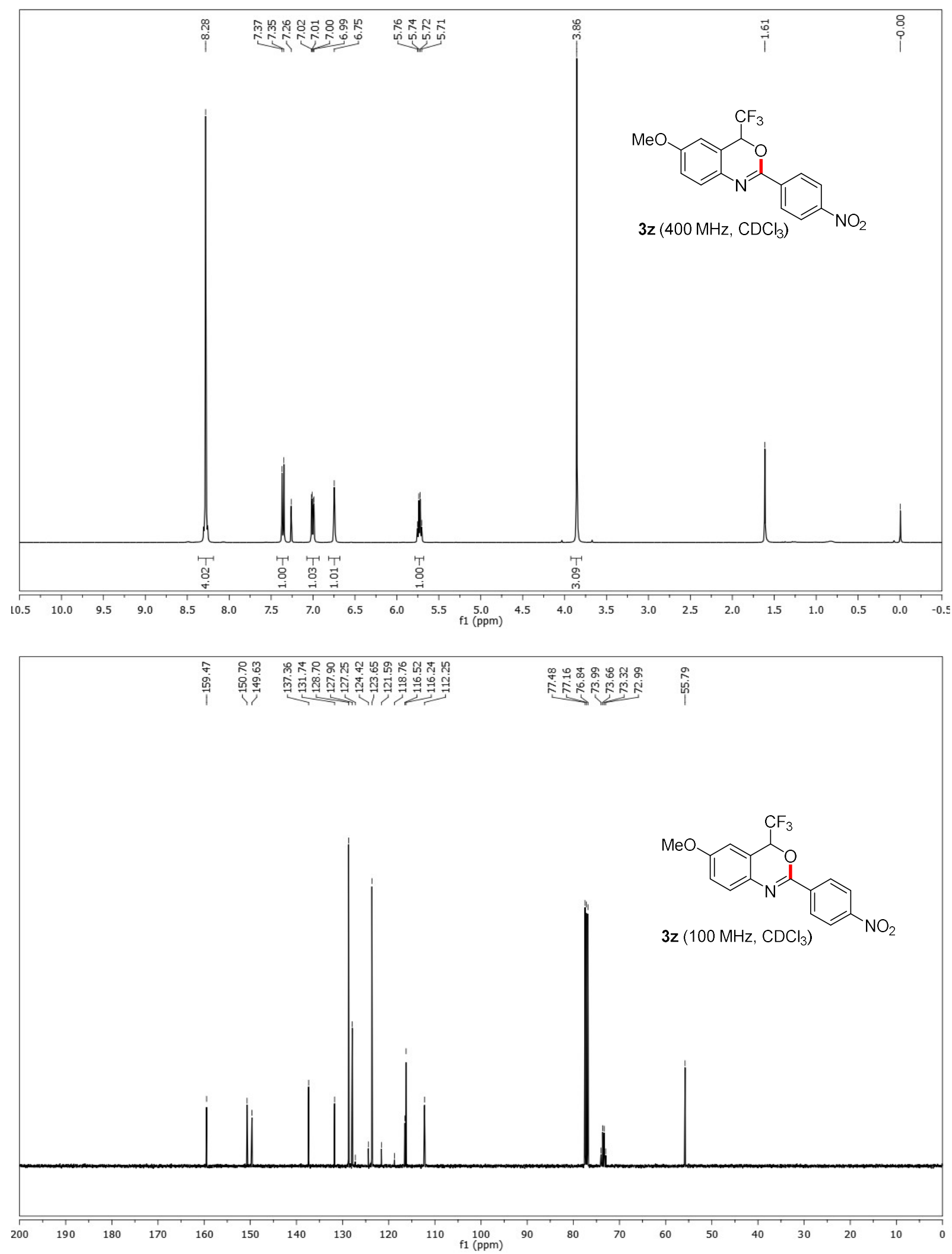
2-(4-Nitrophenyl)-4-(trifluoromethyl)-4H-benzo[d][1,3] oxazin-6-yl benzoate (3aa)
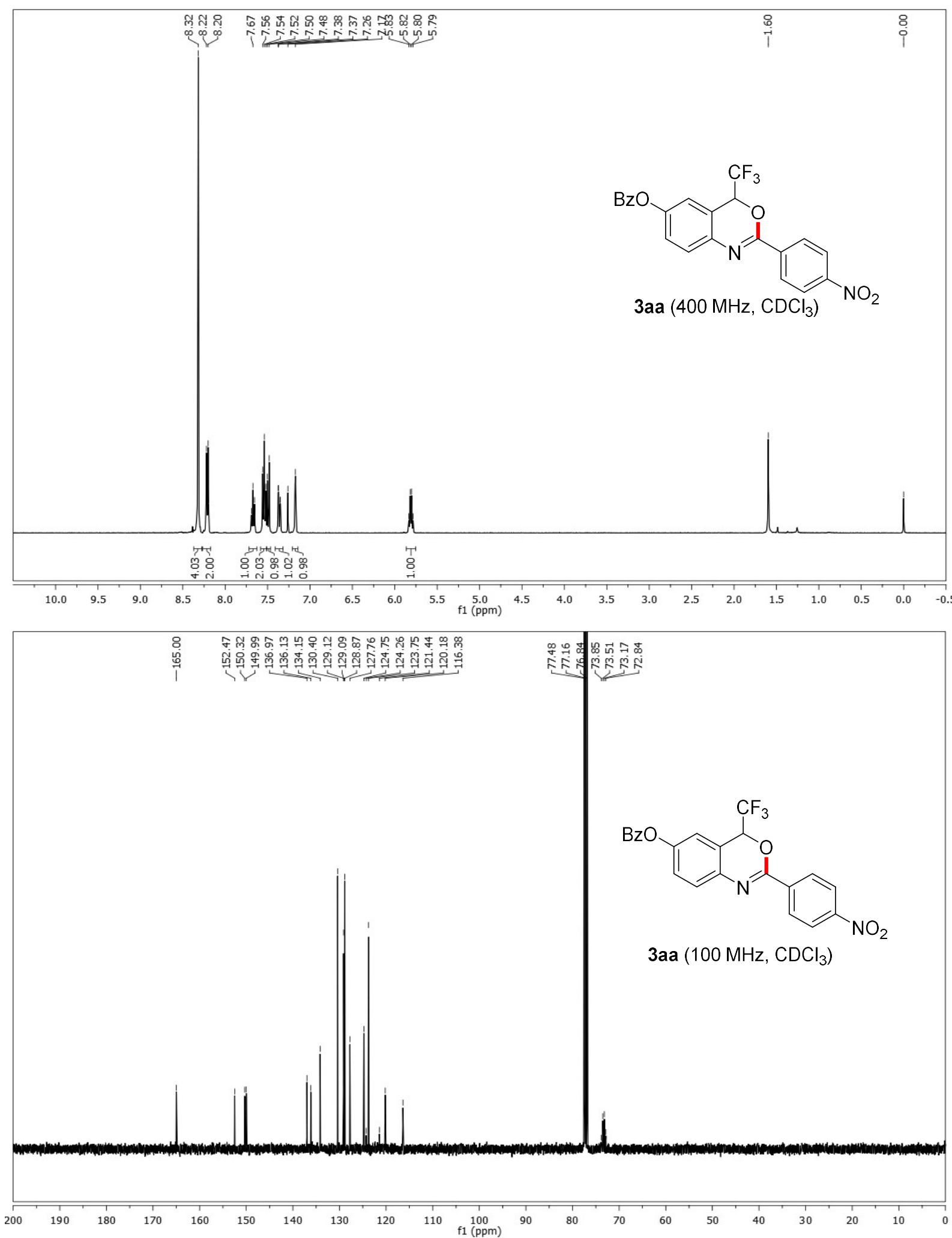
Methyl 2-(4-nitrophenyl)-4-(trifluoromethyl)-4H-benzo[d] [1,3]oxazine-7-carboxylate (3ab)
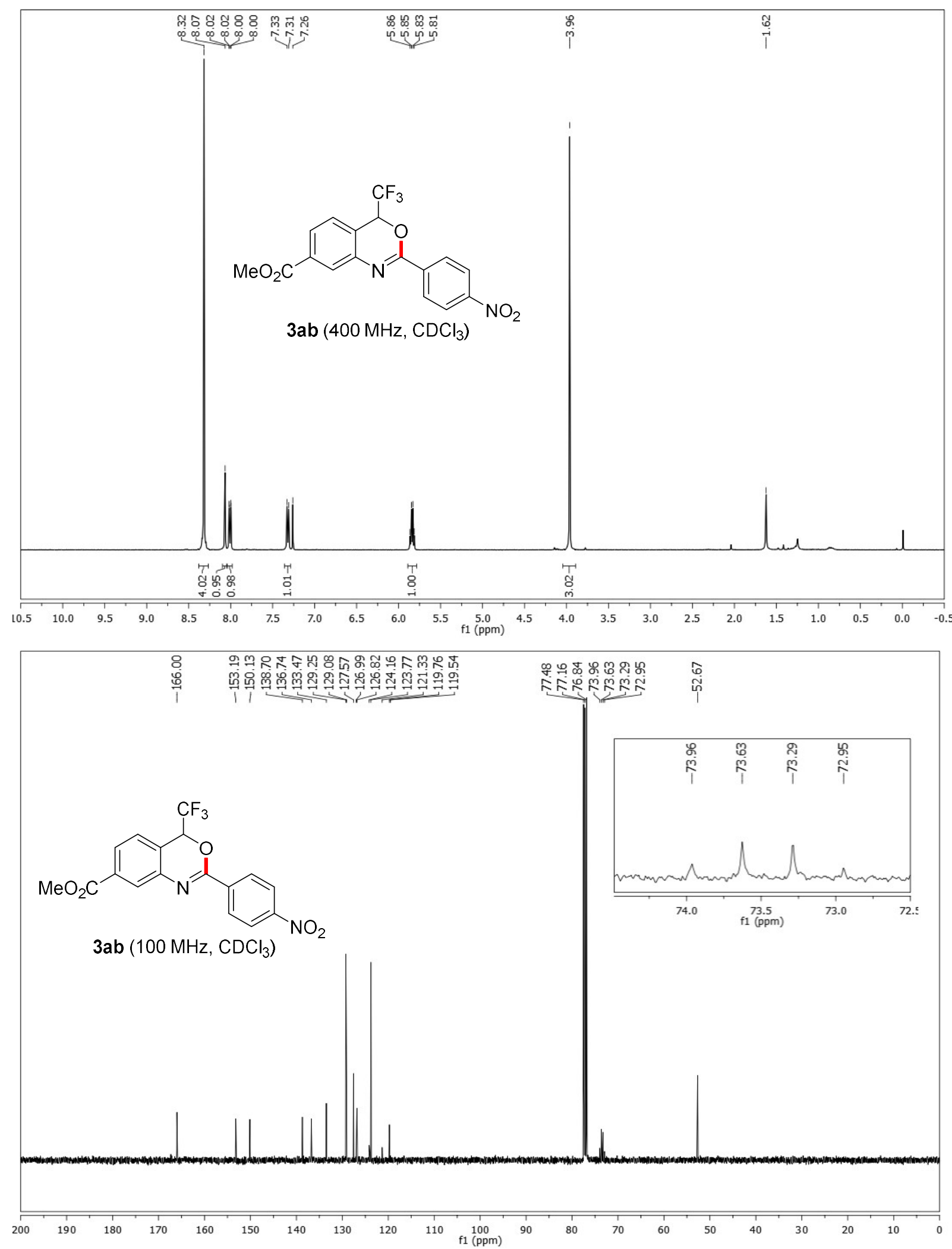
4-Methyl-2-(4-nitrophenyl)-4-(trifluoromethyl)-4H-benzo[d][1,3]oxazine (3ac)
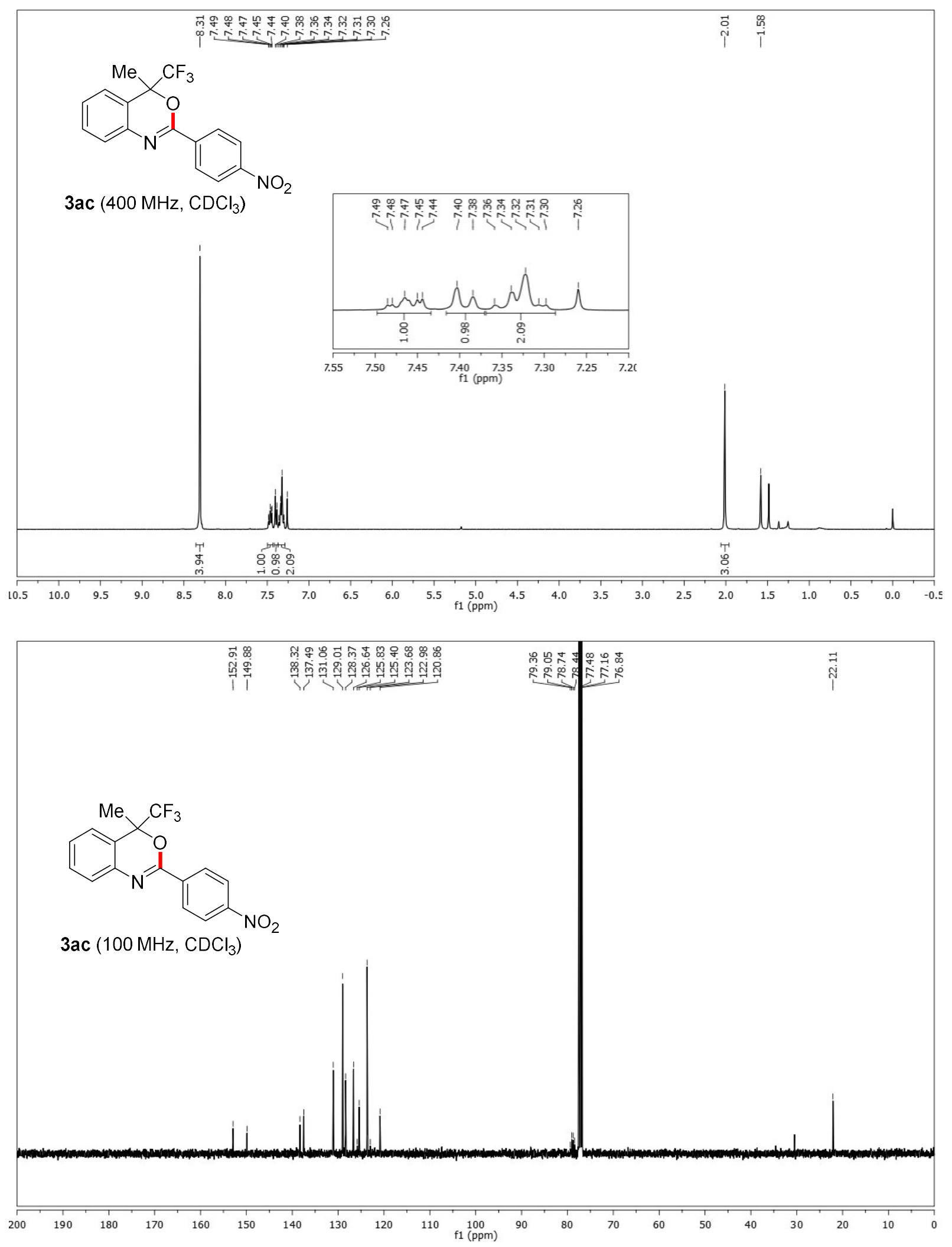

S43 
2-(4-Nitrophenyl)-4,4-bis(trifluoromethyl)-4H-benzo $[d][1,3]$ oxazine (3ad)
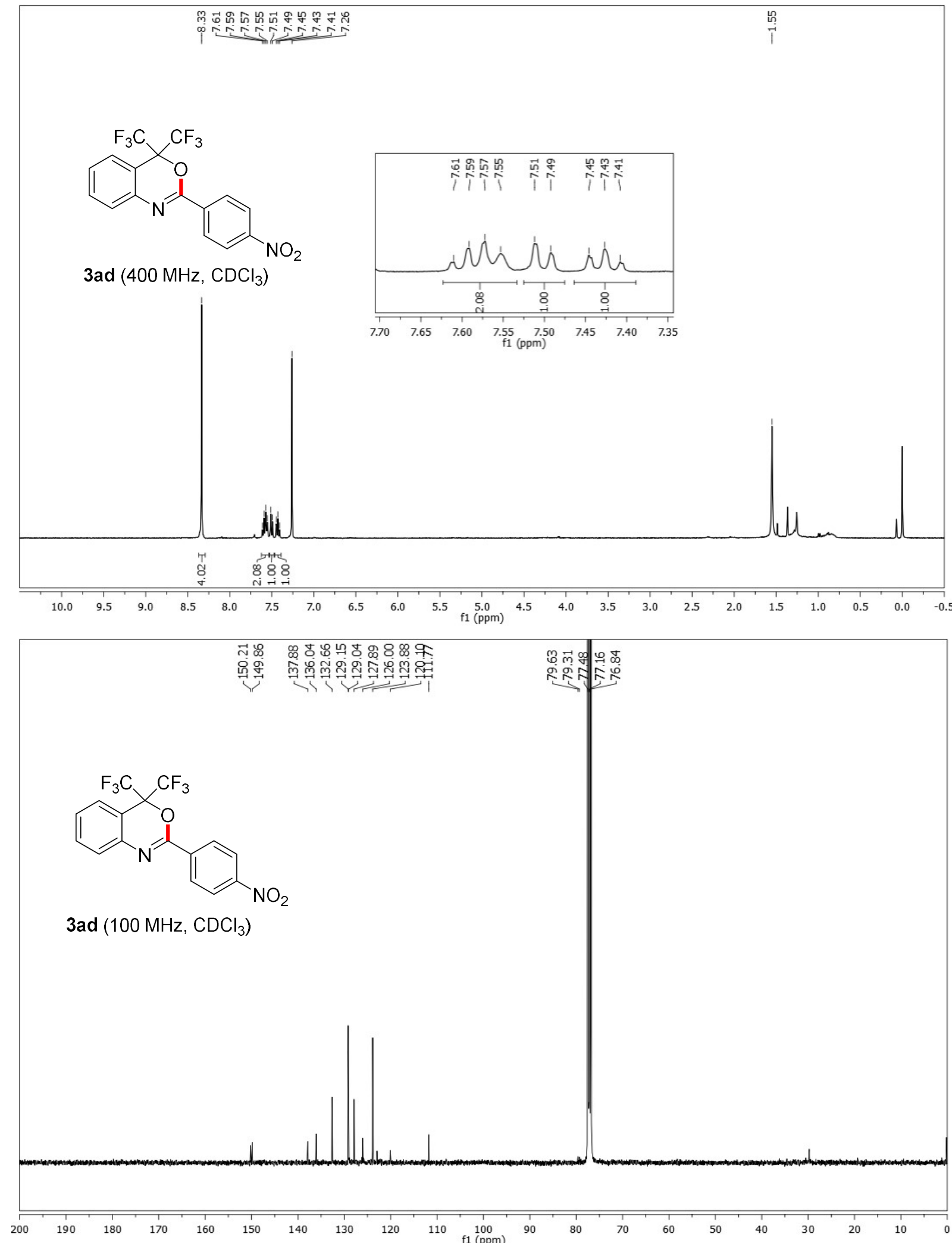


\section{Cartesian Coordinates for the Optimized Structures}

\section{Imine 4a}

\begin{tabular}{|c|c|c|c|}
\hline $\mathrm{C}$ & 0.74917100 & 3.12490300 & -0.25830700 \\
\hline $\mathrm{C}$ & 0.37525900 & 2.28804500 & 0.78884200 \\
\hline $\mathrm{C}$ & 1.01502500 & 1.05717200 & 0.96213100 \\
\hline $\mathrm{C}$ & 2.04916800 & 0.66904800 & 0.09326500 \\
\hline $\mathrm{C}$ & 2.40443300 & 1.51521100 & -0.95803400 \\
\hline $\mathrm{C}$ & 1.76223600 & 2.74009000 & -1.13420900 \\
\hline $\mathrm{H}$ & 0.24909600 & 4.07985000 & -0.38701100 \\
\hline $\mathrm{H}$ & -0.40711100 & 2.57895400 & 1.48395500 \\
\hline $\mathrm{H}$ & 3.19002800 & 1.22962200 & -1.64868900 \\
\hline $\mathrm{H}$ & 2.05637100 & 3.38900000 & -1.95221700 \\
\hline $\mathrm{C}$ & 2.65091600 & -0.69377000 & 0.33764600 \\
\hline $\mathrm{H}$ & 2.89739400 & -0.78086800 & 1.40494200 \\
\hline $\mathrm{C}$ & 3.95094300 & -0.96324400 & -0.40971300 \\
\hline $\mathrm{F}$ & 4.44063000 & -2.16732100 & -0.05161100 \\
\hline $\mathrm{F}$ & 3.80003300 & -0.99177100 & -1.74266400 \\
\hline $\mathrm{F}$ & 4.89186100 & -0.04960600 & -0.12367100 \\
\hline O & 1.70534500 & -1.67620400 & -0.03893300 \\
\hline $\mathrm{H}$ & 1.90808200 & -2.50973800 & 0.40692900 \\
\hline $\mathrm{N}$ & 0.71408200 & 0.24560800 & 2.07707200 \\
\hline $\mathrm{C}$ & -0.43019300 & -0.28438600 & 2.24734300 \\
\hline $\mathrm{C}$ & -1.60375400 & -0.29506600 & 1.33249900 \\
\hline $\mathrm{C}$ & -2.87711600 & -0.28759600 & 1.91427000 \\
\hline $\mathrm{C}$ & -1.47295400 & -0.37316000 & -0.06097700 \\
\hline $\mathrm{C}$ & -4.01924500 & -0.31054600 & 1.12227500 \\
\hline $\mathrm{H}$ & -2.97498100 & -0.25644100 & 2.99575300 \\
\hline $\mathrm{C}$ & -2.60520700 & -0.41424100 & -0.86548700 \\
\hline $\mathrm{H}$ & -0.48920400 & -0.42865200 & -0.51494200 \\
\hline $\mathrm{C}$ & -3.85418800 & -0.37084100 & -0.25548000 \\
\hline $\mathrm{H}$ & -5.01256000 & -0.29039800 & 1.55388500 \\
\hline $\mathrm{H}$ & -2.52822100 & -0.48292800 & -1.94388100 \\
\hline $\mathrm{N}$ & -5.05537600 & -0.40644800 & -1.10608200 \\
\hline O & -4.89662000 & -0.44266900 & -2.31398700 \\
\hline O & -6.14311600 & -0.39698300 & -0.55527800 \\
\hline $\mathrm{H}$ & -0.58438900 & -0.80710000 & 3.1 \\
\hline
\end{tabular}

\section{Imidoyl azolium A}

$\begin{array}{llll}\mathrm{C} & -2.35760200 & -2.32564500 & 2.87331700 \\ \mathrm{C} & -1.63658600 & -1.21191200 & 2.45199000 \\ \mathrm{C} & -1.95997500 & -0.59957500 & 1.23955200 \\ \mathrm{C} & -3.00830600 & -1.08706900 & 0.44168000 \\ \mathrm{C} & -3.70294400 & -2.21824300 & 0.86966500 \\ \mathrm{C} & -3.38551000 & -2.83123100 & 2.08137100 \\ \mathrm{H} & -2.11315300 & -2.79708200 & 3.81975200 \\ \mathrm{H} & -0.83600100 & -0.80317600 & 3.06182600 \\ \mathrm{H} & -4.50415400 & -2.63112500 & 0.26757500 \\ \mathrm{H} & -3.94511600 & -3.70306100 & 2.40275000\end{array}$


$-0.36984900$

0.70251900

$-0.80409800$

$-0.05606100$

$-2.08332200$

$-0.62905100$

$-0.58053800$

0.00465000

0.58535800

0.63088500

$-0.49434900$

$-1.67545400$

$-0.33862400$

$-2.69487500$

$-1.77871100$

$-1.35084600$

0.57853300

$-2.50201900$

$-3.61240600$

$-1.25111300$

$-3.56199200$

$-3.35934100$

$-4.57202600$

2.00027000

2. 41097700

3.12523900

1.61945100

1.86662700

0.62465300

1.05974300

2.66239800

$-0.18685700$

0.47870700

0.02602900

1.23151600

$-0.98089500$

$-0.61016800$

3.76898600

4.17729200

3.53381200

3.37982000

2. 92919300

5.02362000

5.62099000

5.11757500

5.47508000

5.88101500

6.21110600
$-0.86474400$

$-0.66097600$

$-1.60208000$

$-2.71340700$

$-1.99645100$

$-0.85211000$

$-1.70831500$

$-2.47659400$

0.82673500

0.64321200

0.67898300

$-0.00848600$

1. 32084300

$-0.07798900$

$-0.51948900$

1.25778400

1.85579600

0.54853600

$-0.61848900$

1.73341100

0.44298100

0.96630500

$-0.16810100$

0.31939800

$-0.36514200$

0.69722800

$-1.00431300$

$-0.67032500$

$-1.90091200$

$-1.23200600$

0.02526700

$-2.43967000$

$-2.16342300$

$-2.10162400$

$-0.98128100$

$-3.12783200$

$-2.52471700$

$-0.41721600$

0.23780400

1.43041700

0.77639500

2.32929900

1.70390600

1.70649200

2.68233600

0.60124100

$-0.27617200$

0.94927400 